\title{
ON DIFFUSION PROCESSES WITH DRIFT IN $L_{d}$
}

\author{
N.V. KRYLOV
}

\begin{abstract}
We investigate properties of Markov quasi-diffusion processes corresponding to elliptic operators $L=a^{i j} D_{i j}+b^{i} D_{i}$, acting on functions on $\mathbb{R}^{d}$, with measurable coefficients, bounded and uniformly elliptic $a$ and $b \in L_{d}\left(\mathbb{R}^{d}\right)$. We show that each of them is strong Markov with strong Feller transition semigroup $T_{t}$, which is also a continuous bounded semigroup in $L_{d_{0}}\left(\mathbb{R}^{d}\right)$ for some $d_{0} \in(d / 2, d)$. We show that $T_{t}, t>0$, has a kernel $p_{t}(x, y)$ which is summable in $y$ to the power of $d_{0} /\left(d_{0}-1\right)$. This leads to the parabolic Aleksandrov estimate with power of summability $d_{0}$ instead of the usual $d+1$. For the probabilistic solution, associated with such a process, of the problem $L u=f$ in a bounded domain $D \subset \mathbb{R}^{d}$ with boundary condition $u=g$, where $f \in L_{d_{0}}(D)$ and $g$ is bounded, we show that it is Hölder continuous. Parabolic version of this problem is treated as well. We also prove Harnack's inequality for harmonic and caloric functions associated with such a process. Finally, we show that the probabilistic solutions are $L_{d_{0}}$-viscosity solutions.
\end{abstract}

\section{INTRODUCTION}

Let $\mathbb{R}^{d}$ be a Euclidean space of points $x=\left(x^{1}, \ldots, x^{d}\right)$. For a fixed throughout the article $\delta \in(0,1)$ define $\mathbb{S}_{\delta}$ as the set of $d \times d$ symmetric matrices whose eigenvalues are between $\delta$ and $\delta^{-1}$. Fix a constant $\|b\| \in(0, \infty)$. In this article we consider and discuss only uniformly nondegenerate processes with bounded diffusion coefficient.

Assumption 1.1. We are given a Borel measurable $\mathbb{S}_{\delta}$-valued function $a=$ $a(x)$ and a Borel measurable $\mathbb{R}^{d}$-valued function $b=b(x)$ such that

$$
\|b\|_{L_{d}\left(\mathbb{R}^{d}\right)} \leq\|b\| .
$$

Define

$$
D_{i}=\frac{\partial}{\partial x^{i}}, \quad D_{i j}=D_{i} D_{j}, \quad L=(1 / 2) a^{i j}(x) D_{i j}+b^{i}(x) D_{i} .
$$

The goal of this article is to investigate (time-homogeneous Markov) quasi-diffusion processes corresponding to $L$. In the more modern terminology from [19] these are called diffusion processes, but at this point and later on we will follow the terminology from [5] in which the notion of diffusion processes is defined differently from [19].

2010 Mathematics Subject Classification. 60J60, 60J35.

Key words and phrases. Itô equations, Markov processes, diffusion processes. 
The definition of time-homogeneous diffusion processes first appeared in the book by Dynkin in 1963, [5], where he also constructs diffusion processes corresponding to elliptic operators as in (1.1) with bounded and Hölder continuous coefficients, such that the matrix $\left(a_{i j}(x)\right)$ is uniformly strictly positive.

If $x_{t}(x), t \geq 0$, is a family of continuous processes on $\mathbb{R}^{d}$, parametrized by $x \in \mathbb{R}^{d}$, and the family is a diffusion process corresponding to the above $L$ in Dynkin's sense, then, for any bounded domain $D \subset \mathbb{R}^{d}$ and smooth function $u$,

$$
u(x)=E_{x}\left[u\left(x_{\tau_{D}}\right)-\int_{0}^{\tau_{D}} L u\left(x_{t}\right) d t\right],
$$

where $\tau_{D}=\tau_{D}(x)$ is the first exit time of $x_{t}(x)$ from $D$.

The author took the above property as the definition of quasi-diffusion process and in 1966 constructed such process under the assumptions that the matrix $\left(a_{i j}(x)\right)$ is uniformly strictly positive, is continuous and $b$ is Borel bounded. The domain of definition of the corresponding generator of the constructed process was also described, which provides the so-called weak uniqueness of the process with this generator. Later on in 1973, ([7]), when Itô's formula was extended to $W_{p}^{2}$ functions, it became obvious that the quasi-diffusion processes corresponding to the operators satisfying the above mentioned condition are weakly unique.

Two years earlier Tanaka constructed Dynkin's diffusion processes when $b$ is also continuous. No uniqueness was implied in his paper.

Quasi-diffusion processes are characterized by the property that, for any smooth function $u(x)$ and starting point $x$, the process

$$
u\left(x_{t}(x)\right)-\int_{0}^{t} L u\left(x_{s}(x)\right) d s
$$

is a local martingale. Stroock and Varadhan (1969), [19], took the timeinhomogeneous version of this property as the definition of diffusion process and proved existence and weak uniqueness under the condition that $a, b$ are bounded, $a$ is uniformly continuous in $x$ uniformly in $t$, and $a$ is uniformly nondegenerate. The proof of uniqueness is based on the solvability of parabolic equations with coefficients depending only on time, a result borrowed from PDE, and the estimate

$$
E_{s, x} \int_{0}^{1} f\left(s+t, x_{t}\right) d t \leq N\left(\int_{0}^{1} \int_{\mathbb{R}^{d}}|f(s+t, y)|^{p} d t d x\right)^{1 / p},
$$

which is achieved by a quite clever argument. This argument, however, is heavily based on the uniform continuity of $a$ with respect to $x$.

In 1974 [8] the author proved that estimate (1.2) holds true not only for solutions of stochastic equations but also in the case that $a, b$ are any progressively measurable bounded functions such that $a$ is uniformly nondegenerate. The method of proof is different from the one used by Stroock and Varadhan (and the range of $p$ is more restrictive). 
Recall that the first quasi-diffusion strong Markov processes with bounded Borel $b$ and Borel uniformly nondegenerate $a$ were constructed in [7]. This construction was carried over to the case of time-inhomogeneous processes with jumps in [1]. A different approach again when $b$ is bounded, based on Krylov-Safonov estimates, is carried out in [2] and produced a particular strong Markov process with strong Feller resolvent.

It is worth mentioning that with sufficiently regular diffusion matrix $a$ and the drift which is a generalized function of the type of the derivative of a measure generalized diffusion processes are constructed in [16]. The case of time-dependent regular $a$ and $b$ summable to powers which are different in $t$ and $x$ attracted a very extensive attention. In that regard the reader can consult [21], [16], and references therein. Our main emphasis here is on

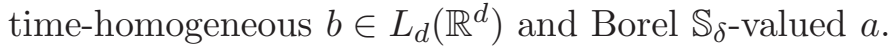

In case $b \in L_{d}$ we established in [13] the existence of a strong Markov quasi-diffusion process corresponding to $L$. Our goal in this article is to investigate properties of just Markov quasi-diffusion processes corresponding to $L$ regardless of how they appeared or were constructed.

In particular, we show that each of them is strong Markov with strong Feller transition semigroup $T_{t}$, which is also a continuous bounded semigroup in $L_{p}\left(\mathbb{R}^{d}\right)$ for $p \in\left[d_{0}, d\right)$, where $d_{0} \in(d / 2, d)$. Our estimate (4.11) implies that $T_{t}$ has a kernel $p_{t}(x, y)$ which is summable with respect to $y$ to the power of $d_{0} /\left(d_{0}-1\right)$. This leads to the parabolic Aleksandrov estimate with power of summability $d_{0}$ instead of usual $d+1$ (see Corollary 4.9 for the probabilistic version and Theorem 4.13 for the PDE version).

For the probabilistic solution, associated with such a process, of the problem $L u+f=0$ in a bounded domain $D \subset \mathbb{R}^{d}$ with boundary condition $u=g$, where $f \in L_{d_{0}}(D)$ and $g$ is Borel bounded, we show that it is Hölder continuous in $D$. Parabolic version of this problem is treated as well. We also prove Harnack's inequality for harmonic and caloric functions associated with such a process. Finally we show that probabilistic solutions of the corresponding elliptic equations are $L_{d_{0}}$-viscosity solutions.

In our arguments self-similar transformations play a very important role. Observe that under such transformations $a$ and $b$ change, but the new $a$ is still in $\mathbb{S}_{\delta}$ and the $L_{d}\left(\mathbb{R}^{d}\right)$-norm of the new $b$ is majorated by the same number $\|b\|$.

We finish the introduction with some notation. For $T, R>0,(t, x) \in$ $\mathbb{R}^{d+1}=\left\{(t, x): t \in \mathbb{R}, x \in \mathbb{R}^{d}\right\}$ define

$$
\begin{gathered}
B_{R}(x)=\left\{y \in \mathbb{R}^{d}:|y-x|<R\right\}, \quad B_{R}=B_{R}(0), \quad C_{T, R}=[0, T) \times B_{R}, \\
C_{T, R}(t, x)=(t, x)+C_{T, R}, \quad C_{R}(t, x)=C_{R^{2}, R}(t, x), \quad C_{R}=C_{R}(0,0), \\
\partial_{t}=\partial / \partial t .
\end{gathered}
$$

In the proofs of various results we use the symbol $N$ to denote finite nonnegative constants which may change from one occurrence to another and we do not always specify on which data these constants depend. In 
these cases the reader should remember that, if in the statement of a result there are constants called $N$ which are claimed to depend only on certain parameters, then in the proof of the result the constants $N$ also depend only on the same parameters unless specifically stated otherwise. Of course, if we write $N=N(\ldots)$, this means that $N$ depends only on what is inside the parentheses. Another point is that when we say that certain constants depend only on such and such parameters we mean, in particular, that the dependence is such that these constants stay bounded as the parameters vary in compact subsets of their ranges.

\section{Diffusions and Itô stochastic Equations}

Suppose that we are given a a quasi-diffusion process corresponding to $L$, that is, we are given a continuous Markov process $X=\left(x_{t}, \infty, \mathcal{M}_{t}, P_{x}\right)$ (the terminology taken from [5]) in $\mathbb{R}^{d}$ such that for any $x \in \mathbb{R}^{d}$ and $t \geq 0$

$$
E_{x} \int_{0}^{t}\left|b\left(x_{s}\right)\right| d s<\infty
$$

and for any twice continuously differentiable function $u$ with compact support

$$
u(x)=E_{x} u\left(x_{t}\right)-E_{x} \int_{0}^{t} L u\left(x_{s}\right) d s .
$$

Remark 2.1. These requirements would be unrealistic if $b$ were only in $L_{p}$ with $p<d$ (see [13]).

Define $\tau_{R}$ as the first exit time of $x_{t}$ from $B_{R}$ (equal to infinity if $x_{t}$ never exits from $B_{R}$ ). This notation $\tau_{R}$ is used throughout the article.

Denote by $\mathcal{N}_{t}$ the $\sigma$-field in $\Omega$ generated by the events $\left\{\omega: x_{s} \in \Gamma\right\}$ for all $s \leq t$ and Borel $\Gamma \subset \mathbb{R}^{d}$ and let $\overline{\mathcal{N}}_{t}^{x_{0}}$ be the completion of $\mathcal{N}_{t}$ with respect to $\left(\mathcal{N}_{\infty}, P_{x_{0}}\right)$.

Here is a start up result.

Theorem 2.1. For any $x_{0} \in \mathbb{R}^{d}$ there exists a d-dimensional Wiener process $w_{t}$ such that $\left(w_{t}, \overline{\mathcal{N}}_{t}^{x_{0}}\right)$ is a Wiener process and $P_{x_{0}}$-(a.s.) for all $t \geq 0$

$$
x_{t}=x_{0}+\int_{0}^{t} \sqrt{a\left(x_{s}\right)} d w_{s}+\int_{0}^{t} b\left(x_{s}\right) d s .
$$

This theorem would be a simple consequence of Theorem 4.5.1 of [19] if $b$ were supposed to be bounded. In our case Theorem 2.1 is a direct corollary of Lemma 1.6 of [13] and the following.

Lemma 2.2. For any $x_{0} \in \mathbb{R}^{d}$ and any twice continuously differentiable function $u$ with compact support the process

$$
\xi_{t}:=u\left(x_{t}\right)-\int_{0}^{t} L u\left(x_{s}\right) d s
$$

is a martingale with respect to $\mathcal{N}_{t}$ and measure $P_{x_{0}}$. 
Proof. What we are given and the Markov property imply that for any $0=t_{0}<t_{1}<\ldots<t_{n}=s<t$ and Borel bounded $g\left(y_{0}, \ldots, y_{n}\right)$ on $\mathbb{R}^{d(n+1)}$

$$
\begin{gathered}
E_{x_{0}} g\left(x_{t_{0}}, \ldots, x_{t_{n}}\right)\left(\xi_{t}-\xi_{s}\right) \\
=E_{x_{0}} g\left(x_{t_{0}}, \ldots, x_{t_{n}}\right)\left(E_{x_{s}} u\left(x_{t-s}\right)-u\left(x_{s}\right)-E_{x_{s}} \int_{0}^{t-s} L u\left(x_{r}\right) d r\right)=0 .
\end{gathered}
$$

It follows that

$$
E_{x_{0}}\left(\xi_{t}-\xi_{s} \mid \overline{\mathcal{N}}_{s}^{x_{0}}\right)=0
$$

which shows that $\left(\xi_{t}, \mathcal{N}_{t}^{x_{0}}\right), t \geq 0$, is, indeed, a martingale.

\section{Some RESUlts FROM [12] AND [13]}

Theorem 2.1 allows us to use the results from [12], [13] some of which we list here.

We need a part of Corollary 1.2 of [12] which we state as follows.

Theorem 3.1. For $0 \leq s<t<\infty, x \in \mathbb{R}^{d}$, and $n \geq 0$ we have

$$
E_{x} \max _{r \in[s, t]}\left|x_{r}-x_{s}\right|^{2 n} \leq N(t-s)^{n}
$$

where $N=N(n, d, \delta,\|b\|)$.

Here are Theorems 2.7 and 2.8 of [13], in which $d_{0}=d_{0}(d, \delta,\|b\|) \in$ $(d / 2, d)$ and for Borel $\Gamma \subset \mathbb{R}^{d}$

$$
\phi_{t}(\Gamma)=\int_{0}^{t} I_{\Gamma}\left(x_{s}\right) d s
$$

Theorem 3.2. Let $p \geq d_{0}$ and $R \in(0, \infty)$. Then there exists $N$, depending only on $p, d, \delta$, and $\|b\|$, such that for any Borel nonnegative $f$ on $\mathbb{R}^{d}$ we have

$$
E_{0} \int_{0}^{\tau_{R}} f\left(x_{t}\right) d t \leq N R^{2-d / p}\|f\|_{L_{p}\left(B_{R}\right)} .
$$

Theorem 3.3. Let $p \geq d_{0}$. Then there exist constants $N$ and $\mu>0$, depending only on $d, p$, and $\|b\|$, and there exists $R_{0}=R_{0}(d,\|b\|) \geq 2$, such that for any $\lambda>0, R \in[0, \infty)$, and Borel nonnegative $f$ given on $\mathbb{R}^{d}$ we have

$$
E_{0} \int_{0}^{\infty} e^{-\lambda \phi_{t}\left(B_{R}^{c}\right)} f\left(x_{t}\right) d t \leq N\left(R \sqrt{\lambda}+R_{0}\right)^{2-d / p} \lambda^{d /(2 p)-1}\left\|\Psi_{R, \lambda}^{-1} f\right\|_{L_{p}\left(\mathbb{R}^{d}\right)},
$$

where $\Psi_{R, \lambda}(x)=\exp \left(\sqrt{\lambda} \mu \operatorname{dist}\left(x, B_{R+R_{0} / \sqrt{\lambda}}\right)\right)$.

Of the same spirit is the following particular case of Theorem 4.7 of [12].

Theorem 3.4. There exists constants $N$ and $\mu>0$, depending only on $d, \delta$, and $\|b\|$, such that for any $\lambda>0$ and Borel nonnegative $f$ given on $\mathbb{R}^{d+1}$ we have

$$
E_{0} \int_{0}^{\infty} e^{-\lambda t} f\left(t, x_{t}\right) d t \leq N \lambda^{-d /(2 d+2)}\left\|\Psi_{\lambda}^{-1} f\right\|_{L_{d+1}\left(\mathbb{R}^{d+1}\right)},
$$

where $\Psi_{\lambda}(x)=\exp (\sqrt{\lambda} \mu|x|)$. 
Introduce

$$
R_{\lambda} f(x)=E_{x} \int_{0}^{\infty} e^{-\lambda t} f\left(x_{t}\right) d t .
$$

Corollary 3.5. Let $q \geq p \geq d_{0}$. Then there exists a constant $N$, depending only on $d, p, q, \delta$, and $\|b\|$, such that for any $\lambda>0$ and Borel nonnegative $f$ given on $\mathbb{R}^{d}$ we have

$$
\begin{gathered}
R_{\lambda} f \leq N \lambda^{d /(2 p)-1}\left\|\Psi_{0, \lambda}^{-1} f\right\|_{L_{p}\left(\mathbb{R}^{d}\right)}, \\
\left\|R_{\lambda} f\right\|_{L_{q}\left(\mathbb{R}^{d}\right)} \leq N \lambda^{-1+(d / 2)(1 / p-1 / q)}\|f\|_{L_{p}\left(\mathbb{R}^{d}\right)} .
\end{gathered}
$$

Indeed, (3.5) is a particular case of (3.3) when $R=0$, which can be rewritten as

$$
\left(R_{\lambda} f(x)\right)^{p} \leq N \lambda^{d / 2-p} \int_{\mathbb{R}^{d}} e^{-\sqrt{\lambda} \mu|x-y|} f^{p}(y) d y .
$$

On the right we have the convolution of two functions. Hence,

$$
\left\|R_{\lambda}^{p} f\right\|_{L_{q / p}\left(\mathbb{R}^{d}\right)} \leq N \lambda^{d / 2-p}\left(\int_{\mathbb{R}^{d}} e^{-\sqrt{\lambda} \mu|x| q / p} d x\right)^{p / q}\left\|f^{p}\right\|_{L_{1}\left(\mathbb{R}^{d}\right)},
$$

which immediately yields (3.6).

Corollary 3.6. Let $p \geq d_{0}$. Then for any $f \in L_{p}\left(\mathbb{R}^{d}\right)$ we have

$$
\lim _{\lambda \rightarrow \infty}\left\|\lambda R_{\lambda} f-f\right\|_{L_{p}\left(\mathbb{R}^{d}\right)}=0 .
$$

Indeed, owing to Corollary 3.5 it suffices to prove (3.7) for $f \in C_{0}^{\infty}\left(\mathbb{R}^{d}\right)$. For such $f$ by Itô's formula (see Theorem 1.3 in [13])

$$
E_{x} f\left(x_{t}\right) e^{-t}=f(x)+E_{x} \int_{0}^{t} e^{-\lambda s}(g-\lambda f)\left(x_{s}\right) d s,
$$

where $g=L f \in L_{d_{0}}\left(\mathbb{R}^{d}\right)$. Hence

$$
\lambda R_{\lambda} f-f=R_{\lambda} g
$$

and owing to (3.6)

$$
\left\|\lambda R_{\lambda} f-f\right\|_{L_{p}\left(\mathbb{R}^{d}\right)} \leq N \lambda^{-1+(d / 2)\left(1 / d_{0}-1 / p\right)}\|g\|_{L_{d_{0}}\left(\mathbb{R}^{d}\right)} .
$$

Here the exponent of $\lambda$ is strictly less than zero because $d_{0}>d / 2$ and this yields (3.7).

We are also going to need Corollary 4.3 of [13], which we state as follows.

Theorem 3.7. For any $R \in(0, \infty)$ and $\kappa \in(0,1)$ there exist constants $\mu \geq 1, \theta>0$, and $N$, depending only on $d, \delta,\|b\|$, and $\kappa$, such that, for any $x \in B_{\kappa R}$ and Borel set $\Gamma \subset B_{R}$

$$
P_{x}\left(\phi_{\tau_{R}}(\Gamma) \geq \theta \gamma^{\mu} R^{2}\right) \geq N^{-1} \gamma^{2 \mu}
$$

where $\gamma=|\Gamma| /\left|B_{R}\right|$.

Here is a specification of Theorem 4.4 of [12] to our case. Recall that

$$
C_{T, R}=[0, T) \times B_{R}, \quad C_{R}=C_{R^{2}, R}
$$


Theorem 3.8. For any $\kappa \in(0,1)$ there is a function $q(\gamma), \gamma \in(0,1)$, depending only on $d, \delta,\|b\|, \kappa$, and, naturally, on $\gamma$, such that for any $R \in$ $(0, \infty), x \in B_{\kappa R}$, and closed $\Gamma \subset C_{R^{2}, R}$ satisfying $|\Gamma| \geq \gamma\left|C_{R^{2}, R}\right|$ we have

$$
P_{x}\left(\tau_{\Gamma} \leq \tau_{R^{2}, R}\right) \geq q(\gamma)
$$

where $\tau_{\Gamma}$ is the first time the process $\left(t, x_{t}\right)$ hits $\Gamma$ and $\tau_{R^{2}, R}$ is its first exit time from $C_{R^{2}, R}$. Furthermore, $q(\gamma) \rightarrow 1$ as $\gamma \uparrow 1$.

Here is a corollary of estimate (2.19) of [12]:

$$
P_{0}\left(\tau_{R} \leq t\right) \leq 2 \exp \left(-\frac{\beta R^{2}}{t}\right)
$$

where $\beta=\beta(d, \delta,\|b\|)>0$.

Here is a corollary of Theorem 4.17 of [13].

Theorem 3.9. Let $R \in(0, \infty), \kappa, \eta \in(0,1), x, y \in B_{\kappa R}$, and $\eta^{-1} R^{2} \geq t \geq$ $\eta R^{2}$. Then there exist $N, \nu>0$, depending only on $\kappa, \eta, d, \delta$, and $\|b\|$, such that, for any $\rho \in(0,1]$,

$$
N P_{x}\left(x_{t} \in B_{\rho R}(y), \tau_{R}>t\right) \geq \rho^{\nu} .
$$

\section{Strong Markov and strong Feller properties of $X$}

Here is one of basic results of this section. Its proof would be greatly simplified if we knew that $X$ is strong Markov. Then we would use stopping times. Instead we use randomized stopping.

Theorem 4.1. Let $p \geq d_{0}, \lambda>0$, and $f \in L_{p}\left(\mathbb{R}^{d}\right)$. Then there exist $\alpha \in(0,1)$ and $N$, depending only on $p, d, \delta$, and $\|b\|$, such that

$$
\left|R_{\lambda} f(x)-R_{\lambda} f(y)\right| \leq N \lambda^{(\alpha p+d) /(2 p)-1}\|f\|_{L_{p}\left(\mathbb{R}^{d}\right)}|x-y|^{\alpha} .
$$

The following is an immediate and well-known consequence of just continuity of $R_{\lambda} f(x)$ with respect to $x$ (see, for instance, Theorem I.8.11 in $[3])$.

Corollary 4.2. The process $\left(x_{t}, \bar{N}_{t+}, P_{x}\right)$ is strong Markov.

Note that strong Markov processes $\left(x_{t}, \bar{N}_{t}, P_{x}\right)$ corresponding to $L$ in case $b$ is bounded are constructed in [7] and strong Markov processes $\left(x_{t}, \bar{N}_{t+}, P_{x}\right)$ corresponding to $L$ in case $b$ is bounded are constructed in [2]. We show that any Markov process corresponding to $L$ with $b \in L_{d}$ is strong Markov with respect to $\bar{N}_{t+}$. Theorem 4.1 will be proved after some preparations.

The following technical result is obtained by using Fubini's theorem for $f_{t}$ vanishing for $t \geq T$ and then letting $T \rightarrow \infty$.

Lemma 4.3. (i) Let $\phi_{t}, \Psi_{t}, f_{t}$ be nonnegative Borel functions on $[0, \infty)$ such that $\Psi_{0}=1, \Psi_{t}$ is continuous and decreasing. Then

$$
\int_{0}^{\infty} e^{-\phi_{t}} f_{t} d t=-\int_{0}^{\infty} e^{-\phi_{t}}\left(\int_{t}^{\infty} e^{-\left(\phi_{s}-\phi_{t}\right)} f_{s} d s\right) d \Psi_{t}+\int_{0}^{\infty} e^{-\phi_{t}} \Psi_{t} f_{t} d t .
$$


(ii) Let $f_{t}$ be a nonnegative Borel function on $[0, \infty)$ and let $\phi_{t}$ and $\psi_{t}$ be absolutely continuous on $[0, \infty)$ such that $\phi_{0}=\psi_{0}$. Assume that

$$
\int_{0}^{\infty} e^{-\phi_{t}} f_{t}+\int_{0}^{\infty} e^{-\psi_{t}}\left|\phi_{t}^{\prime}-\psi_{t}^{\prime}\right|\left(\int_{t}^{\infty} e^{-\left(\phi_{s}-\phi_{t}\right)} f_{s} d s\right) d t<\infty
$$

Then

$$
\int_{0}^{\infty} e^{-\phi_{t}} f_{t} d t=\int_{0}^{\infty} e^{-\psi_{t}} f_{t} d t-\int_{0}^{\infty} e^{-\psi_{t}}\left(\phi_{t}^{\prime}-\psi_{t}^{\prime}\right)\left(\int_{t}^{\infty} e^{-\left(\phi_{s}-\phi_{t}\right)} f_{s} d s\right) d t
$$

In the sequel we use the parameter $n$ that will be ultimately send to infinity. Note that by $R_{n}\left(B_{R}\right)$ one usually means the resolvent at $\lambda=n$ of the process killed outside $B_{R}$. Our notation has a different meaning. If $n \rightarrow \infty$, our $R_{n}\left(B_{R}\right)$ converges to the resolvent at $\lambda=0$ of the process killed outside $B_{R}$.

Lemma 4.4. Let $f$ be a nonnegative Borel bounded function on $\mathbb{R}^{d}$. For Borel sets $\Gamma \subset \mathbb{R}^{d}$ define

$$
\phi_{t}(\Gamma)=\int_{0}^{t} I_{\Gamma}\left(x_{s}\right) d s, \quad u(x)=E_{x} \int_{0}^{\infty} e^{-t} f\left(x_{t}\right) d t .
$$

Also for $R>0$ set

$$
R_{n}\left(B_{R}\right) f(x)=E_{x} \int_{0}^{\infty} e^{-n \phi_{t}\left(B_{R}^{c}\right)} f\left(x_{t}\right) d t
$$

Then

$$
u=R_{1}\left(B_{R}\right) f-R_{1}\left(B_{R}\right) I_{B_{R}} u .
$$

Furthermore, for any $n \geq 1$

$$
R_{1}\left(B_{R}\right) f=R_{n}\left(B_{R}\right) f+(n-1) R_{n}\left(B_{R}\right) I_{B_{R}^{c}} R_{1}\left(B_{R}\right) f
$$

Finally, for any Borel $\Gamma \subset B_{R}$ and $h^{n}=n R_{n}\left(B_{R}\right) f$

$$
\begin{aligned}
h^{n}(x) & =E_{x} \int_{0}^{\infty} e^{-n \phi_{t}\left(\Gamma \cup B_{R}^{c}\right)} n I_{\Gamma}\left(x_{t}\right) h^{n}\left(x_{t}\right) d t \\
& +n E_{x} \int_{0}^{\infty} e^{-n \phi_{t}\left(\Gamma \cup B_{R}^{c}\right)} f\left(x_{t}\right) d t .
\end{aligned}
$$

Proof. Equation (4.2) is obtained by using the Markov property of $X$ and by applying Lemma 4.3 (ii) with $\phi_{t}=t, \psi_{t}=\phi_{t}\left(B_{R}^{c}\right)$. To check that Lemma 4.3 (ii) is applicable we use Theorem 3.3 according to which

$$
E_{x} \int_{0}^{\infty} e^{-\phi_{t}\left(B_{R}^{c}\right)} d t<\infty .
$$

Equation (4.3) is obtained directly by using the Markov property and Lemma 4.3 (i) with $\phi_{t}=\phi_{t}\left(B_{R}^{c}\right), \Psi_{t}=\exp \left(-(n-1) \phi_{t}\left(B_{R}^{c}\right)\right)$.

Finally, to get (4.4) it suffices in Lemma 4.3 (i) to take $\phi_{t}=n \phi_{t}\left(B_{R}^{c}\right)$, and $\Psi_{t}=\exp \left(-n \phi_{t}(\Gamma)\right)$. The lemma is proved. 
Remark 4.1. Send $n \rightarrow \infty$ in (4.3) and (4.4) assuming that $f=0$ in $B_{R}$ in (4.4). Then intuitively we should get

$$
\begin{gathered}
u(x):=E_{x} \int_{0}^{\tau_{R}} f\left(x_{t}\right) d t=R_{1}\left(B_{R}\right) f-E_{x} R_{1}\left(B_{R}\right) f\left(x_{\tau_{R}}\right), \\
h^{n}(x) \rightarrow h(x):=E_{x} f\left(x_{\tau_{R}}\right), \quad h(x)=E_{x}\left(I_{\tau_{\Gamma}<\tau_{R}} h\left(x_{\tau_{\Gamma}}\right)+I_{\tau_{\Gamma}>\tau_{R}} f\left(x_{\tau_{R}}\right)\right),
\end{gathered}
$$

where $\tau_{\Gamma}$ is the first time $x_{t}$ hits $\Gamma$. The formulas we get this way are true indeed if we know that $X$ is strong Markov (and that $\tau_{\Gamma}$ makes sense and is a stopping time). Of course, $u$ and $h$ are the objects of main interest, but we cannot handle them because we do not know yet that $X$ is strong Markov. That is why instead of using stopping times we use randomized ones when, for instance in case of (4.3), we stop $x_{t}$ on each time interval $d t$ it spends outside $B_{R}$ with probability $(n-1) d t$ (provided it was not stopped before).

The next two lemmas are aimed at partially justifying what is said in Remark 4.1.

Lemma 4.5. Let $c$ be a nonnegative function such that $c \geq 1$ on $B_{\rho}^{c}$. Then for any $n, \rho, \varepsilon>0$, and $|x|<\rho+\varepsilon$

$$
I_{n, \varepsilon}:=E_{x} \int_{\tau_{\rho+2 \varepsilon}}^{\infty} e^{-n \phi_{t}} n c\left(x_{t}\right) d t \leq 2 e^{-\sqrt{n} \varepsilon / N}
$$

where $N=N(d, \delta,\|b\|)$ and

$$
\phi_{t}=\int_{0}^{t} c\left(x_{s}\right) d s
$$

Proof. We look at the representation of $x_{t}$ as a solution of (2.2) with $x_{0}=x$. Then after defining $\gamma$ as the first time after $\tau_{\rho+\varepsilon}$ the process $x_{t}$ exits from $B_{\varepsilon}\left(x_{\tau_{\rho+\varepsilon}}\right)$ we note that

$$
I_{n, \varepsilon} \leq E_{x} e^{-n \phi_{\tau_{\rho+2 \varepsilon}}} \leq E_{x} E\left(e^{-n\left(\phi_{\gamma}-\phi_{\tau_{\rho+\varepsilon}}\right)} \mid \bar{N}_{\tau_{\rho+\varepsilon}}\right) .
$$

Here, by the conditional version of Theorem 2.10 of [12], the conditional expectation (a.s.) is dominated by $2 e^{-\sqrt{n} \varepsilon / N}$, and this proves the lemma.

Lemma 4.6. For Borel $\Gamma$ and $\rho>0$ define $\Gamma_{\rho}=\Gamma \cap B_{\rho}$. Assume that $\Gamma$ and $\rho$ are such that $\left|\Gamma_{\rho}\right| \geq(1 / 2)\left|B_{\rho}\right|$ and $\Gamma_{3 \rho}=\Gamma_{\rho} \cup\{2 \rho \leq|x|<3 \rho\}$. Then there are constant $N, \nu>0$, depending only on $d, \delta$, and $\|b\|$, such that for any $n>0$ and $|x| \leq(5 / 2) \rho$

$$
1 \geq n E_{x} \int_{0}^{\tau_{3 \rho}} I_{\Gamma_{3 \rho}}\left(x_{t}\right) e^{-n \phi_{t}\left(\Gamma_{3 \rho}\right)} d t \geq 1-2 e^{-\sqrt{n} \rho / N}
$$

and for $|x| \leq \rho$

$$
I:=E_{x} \int_{0}^{\tau_{3 \rho}} n I_{\Gamma_{\rho}}\left(x_{t}\right) e^{-n \phi_{t}\left(\Gamma_{3 \rho}\right)} d t \geq \nu-N e^{-n \rho^{2} / N} .
$$


Proof. Observe that

$$
I \geq E_{x} \int_{0}^{\tau_{2 \rho}} n I_{\Gamma_{\rho}}\left(x_{t}\right) e^{-n \phi_{t}\left(\Gamma_{\rho}\right)} d t=1-E_{x} e^{-n \phi_{\tau_{2 \rho}}\left(\Gamma_{\rho}\right)},
$$

where for $\gamma=\left|\Gamma_{\rho}\right| /\left|B_{2 \rho}\right|\left(\geq 2^{-d-1}\right)$ owing to Theorem 3.7

$$
\begin{gathered}
E_{x} e^{-n \phi_{\tau_{2 \rho}}\left(\Gamma_{\rho}\right)} \leq P\left(\phi_{\tau_{2 \rho}}\left(\Gamma_{\rho}\right) \leq \theta \gamma^{\mu}(2 \rho)^{2}\right) \\
+e^{-n \theta \gamma^{\mu}(2 \rho)^{2}} P\left(\phi_{\tau_{2 \rho}}\left(\Gamma_{\rho}\right) \geq \theta \gamma^{\mu}(2 \rho)^{2}\right) \\
=1-\left(1-e^{-n \rho^{2} / N}\right) P\left(\phi_{\tau_{2 \rho}}\left(\Gamma_{\rho}\right) \geq \theta \gamma^{\mu}(2 \rho)^{2}\right) \leq 1-\left(1-e^{-n \rho^{2} / N}\right) N^{-1} \gamma^{2 \mu} .
\end{gathered}
$$

This proves (4.6).

To prove (4.5) denote by $\gamma$ the first exit time of $x_{t}$ after $\tau_{(5 / 2) \rho}$ from $B_{(1 / 2) \rho}\left(x_{\tau_{(5 / 2) \rho}}\right)$ and observe that

$$
\begin{aligned}
0 \leq 1 & -n E_{x} \int_{0}^{\tau_{3 \rho}} I_{\Gamma_{3 \rho}}\left(x_{t}\right) e^{-n \phi_{t}\left(\Gamma_{3 \rho}\right)} d t=E_{x} e^{-n \phi_{\tau_{3 \rho}}\left(\Gamma_{3 \rho}\right)} \\
& \leq E_{x} E\left(e^{-n\left(\phi_{\gamma}\left(\Gamma_{3 \rho}\right)-\phi_{\tau_{(5 / 2) \rho}}\left(\Gamma_{3 \rho}\right)\right)} \mid \mathcal{F}_{\tau_{(5 / 2) \rho}}\right) .
\end{aligned}
$$

Here, by the conditional version of Theorem 2.10 of [12], the conditional expectation (a.s.) is dominated by $2 e^{-\sqrt{n} \rho / N}$, and this proves the lemma.

Before doing the next almost final step in our preparation to prove Theorem 4.1, take $n \geq 1, R \in(0,1]$, a bounded Borel $g \geq 0$, such that $g(x)=0$ for $|x|<R$ and introduce

$$
h^{n}=n R_{n}\left(B_{R}\right) g
$$

Observe that

$$
h^{n} \leq(\sup g) n E_{x} \int_{0}^{\infty} e^{-n \phi_{t}\left(B_{R}^{c}\right)} I_{B_{R}^{c}}\left(x_{t}\right) d t \leq \sup g .
$$

The proof of the following lemma, actually, is just a simple adaptation of what is usually done in the theory of elliptic equations when they prove the Hölder continuity of harmonic functions associated with elliptic operators. Again lacking the strong Markov property and knowing nothing about the sets $\Gamma^{n}$, introduced below, apart from the fact that they are Borel, forces us to use randomized stopping times. Somewhat cleaner this adaptation is seen in our Section 6. Concerning the origin of our arguments see Section 9.6 in [11].

Lemma 4.7. There exist constants $\alpha \in(0,1)$ and $N$, depending only on $d, \delta$, and $\|b\|$, and there exists a constant $N^{\prime}$, depending only on $d, \delta,\|b\|$, and $\sup g$, such that for $|x| \leq R / 12$ and any $n \geq 1$ we have

$$
\left|h^{n}(x)-h^{n}(0)\right| \leq N(|x| / R)^{\alpha} \sup _{B_{R}} h^{n}+N^{\prime}\left(e^{-n|x|^{2} / N}+e^{-\sqrt{n}|x| / N}\right) .
$$


Proof. For $\rho>0$ introduce the notation

$\underset{B_{\rho}}{\operatorname{Osc}} u=\sup _{B_{\rho}} u-\inf _{B_{\rho}} u, \quad M_{\rho}^{n}=\sup _{B_{\rho}} h^{n}, \quad m_{\rho}^{n}=\inf _{B_{\rho}} h^{n}, \quad \mu_{\rho}^{n}=\left(M_{\rho}^{n}+m_{\rho}^{n}\right) / 2$.

Take $\rho>0$, such that $\rho \leq R / 4$, and consider two cases

(a) $\left|B_{\rho} \cap\left\{h^{n} \geq \mu_{\rho}^{n}\right\}\right| \geq(1 / 2)\left|B_{\rho}\right|$,

(b) $\left|B_{\rho} \cap\left\{h^{n} \leq \mu_{\rho}^{n}\right\}\right| \geq(1 / 2)\left|B_{\rho}\right|$.

In case (a) introduce $\Gamma^{n}=\left(B_{\rho} \cap\left\{h^{n} \geq \mu_{\rho}^{n}\right\}\right) \cup\left(B_{2 \rho}^{c} \cap B_{R}\right)$. By using (4.4) and Lemma 4.5 for $|x| \leq \rho$ we find that

$$
h^{n}(x)=E_{x} \int_{0}^{\tau_{3 \rho}} e^{-n \phi_{t}\left(\Gamma^{n}\right)} n I_{\Gamma^{n}}\left(x_{t}\right) h^{n}\left(x_{t}\right) d t+\xi^{n}(x)=: h_{0}^{n}(x)+\xi^{n}(x),
$$

where $\left|\xi^{n}(x)\right| \leq N^{\prime} e^{-\sqrt{n} \rho / N}$. Furthermore, $h^{n} \geq \mu_{\rho}^{n}$ on $\Gamma_{\rho}^{n}$ and $h^{n} \geq m_{3 \rho}^{n}$ in $B_{3 \rho}$. Hence

$$
\begin{aligned}
h_{0}^{n}(x) & \geq\left(\mu_{\rho}^{n}-m_{3 \rho}^{n}\right) E_{x} \int_{0}^{\tau_{3 \rho}} e^{-n \phi_{t}\left(\Gamma^{n}\right)} n I_{\Gamma_{\rho}^{n}}\left(x_{t}\right) d t \\
& +m_{3 \rho}^{n} E_{x} \int_{0}^{\tau_{3 \rho}} e^{-n \phi_{t}\left(\Gamma^{n}\right)} n I_{\Gamma^{n}}\left(x_{t}\right) d t .
\end{aligned}
$$

It follows by Lemma 4.6 (also note that $\mu_{\rho}^{n}-m_{3 \rho}^{n} \geq 0$ ) that for $|x| \leq \rho$

$$
h_{0}^{n}(x) \geq \nu \mu_{\rho}^{n}+(1-\nu) m_{3 \rho}^{n}-N^{\prime}\left(e^{-n \rho^{2} / N}+e^{-\sqrt{n} \rho / N}\right),
$$

which in light of the arbitrariness of $x$ and (4.8) implies that

$$
(1-\nu / 2) m_{\rho}^{n} \geq(\nu / 2) M_{\rho}^{n}+(1-\nu) m_{3 \rho}^{n}-N^{\prime}\left(e^{-n \rho^{2} / N}+e^{-\sqrt{n} \rho / N}\right) .
$$

On the other hand, obviously

$$
(1-\nu / 2) M_{\rho}^{n} \leq(\nu / 2) M_{\rho}^{n}+(1-\nu) M_{3 \rho}^{n} .
$$

By subtracting the last two inequalities we get

$$
\begin{gathered}
(1-\nu / 2) \underset{B_{\rho}}{\operatorname{osc}} h^{n} \leq(1-\nu) \underset{B_{3 \rho}}{\operatorname{osc}} h^{n}+N^{\prime}\left(e^{-n \rho^{2} / N}+e^{-\sqrt{n} \rho / N}\right), \\
\quad \underset{B_{\rho}}{\operatorname{osc}_{2}} h^{n} \leq \theta \underset{B_{3 \rho}}{\operatorname{osc}} h^{n}+N^{\prime}\left(e^{-n \rho^{2} / N}+e^{-\sqrt{n} \rho / N}\right),
\end{gathered}
$$

where $\theta=\theta(d, \delta,\|b\|)=(1-\nu) /(1-\nu / 2)<1$.

In case (b) introduce $\Gamma^{n}=\left(B_{\rho} \cap\left\{h^{n} \leq \mu_{\rho}^{n}\right\}\right) \cup\left(B_{2 \rho}^{c} \cap B_{R}\right)$. As in case (a), we have (4.9), where $h^{n} \leq \mu_{\rho}^{n}$ on $\Gamma_{\rho}^{n}$ and $h^{n} \leq M_{3 \rho}^{n}$ om $B_{3 \rho}$. Hence,

$$
\begin{aligned}
h_{0}^{n}(x) & \leq\left(\mu_{\rho}^{n}-M_{3 \rho}^{n}\right) E_{x} \int_{0}^{\tau_{3 \rho}} e^{-n \phi_{t}\left(\Gamma^{n}\right)} n I_{\Gamma_{\rho}^{n}}\left(x_{t}\right) d t \\
& +M_{3 \rho}^{n} E_{x} \int_{0}^{\tau_{3 \rho}} e^{-n \phi_{t}\left(\Gamma^{n}\right)} n I_{\Gamma^{n}}\left(x_{t}\right) d t .
\end{aligned}
$$

Here $\mu_{\rho}^{n}-M_{3 \rho}^{n} \leq 0$ and the last expectation is less than one. Then by (4.6)

$$
h_{0}^{n}(x) \leq\left(\mu_{\rho}^{n}-M_{3 \rho}^{n}\right) \nu+M_{3 \rho}^{n}+N^{\prime} e^{-n \rho^{2} / N},
$$


which in light of the arbitrariness of $x$ and (4.8) implies that

$$
(1-\nu / 2) M_{\rho}^{n} \leq(\nu / 2) m_{\rho}^{n}+(1-\nu) M_{3 \rho}^{n}+N^{\prime}\left(e^{-n \rho^{2} / N}+e^{-\sqrt{n} \rho / N}\right) .
$$

On the other, hand obviously

$$
(1-\nu / 2) m_{\rho}^{n} \geq(\nu / 2) m_{\rho}^{n}+(1-\nu) m_{3 \rho}^{n} .
$$

By subtracting the last two inequalities we get (4.9) again.

From (4.9) we see that

$$
\theta^{-k} \underset{B_{3}-k}{\operatorname{osc}} h^{n} \leq \theta^{-k+1} \underset{B_{3}-k+1}{\operatorname{osc}} h^{n}+\theta^{-k} N^{\prime}\left(e^{-n 3^{-2 k} / N}+e^{-\sqrt{n} 3^{-k} / N}\right),
$$

as long as $3^{-k} \leq R / 4$, that is $k \geq\left\lfloor\log _{3}(4 / R)\right\rfloor=: k_{0}$. By observing that, for instance, $\exp \left(-n 3^{-2 k} \rho^{2} / N\right)$ is an increasing function of $k$ we obtain, for $k \geq k_{0}$

$$
\theta^{-k} \underset{B_{3}-k}{\operatorname{osc}} h^{n} \leq \theta^{-k_{0}} \underset{B_{3^{-k}}}{\operatorname{osc}} h^{n}+N^{\prime}\left(e^{-n 3^{-2 k} / N}+e^{-\sqrt{n} 3^{-k} / N}\right) \sum_{i=0}^{k-k_{0}-1} \theta^{-k+i} .
$$

For $|x| \leq R / 12$ and $k=\left\lfloor\log _{3}(1 /|x|)\right\rfloor$ we have

$$
|x| \leq 3^{-k} \leq R / 4, \quad \theta^{-1}|x|^{-\alpha} \geq \theta^{-k} \geq|x|^{-\alpha},
$$

where $\alpha=-\log _{3} \theta$. Furthermore,

$$
3^{-k} \geq|x|, \quad \theta^{-k_{0}} \leq 4^{\alpha} R^{-\alpha} .
$$

Now (4.7) follows. The lemma is proved.

Proof of Theorem 4.1. Self-similarity transformations show that we may assume that $\lambda=1$. Furthermore, obviously we may assume that $f \geq 0$. Estimate (3.3) allows us to assume that $f$ is bounded and continuous. Then take $R \in(0,1], n \geq 2$, and take $g=I_{B_{R}^{c}} R_{1}\left(B_{R}\right) f$ in Lemma 4.7. Observe that by (4.3) we have $h^{n} \leq 2 R_{1}\left(B_{R}\right) f$. Furthermore, in light of (4.3), Lemma 4.7, and Theorem 3.3, for $0<|x| \leq R / 12$ we have

$$
\begin{gathered}
\quad\left|R_{1}\left(B_{R}\right) f(x)-R_{1}\left(B_{R}\right) f(0)\right| \leq N\left(R+R_{0} / \sqrt{n}\right)^{2-d / p}\|f\|_{L_{p}\left(\mathbb{R}^{d}\right)} \\
+N(|x| / R)^{\alpha}\left(R+R_{0}\right)^{2-d / p}\|f\|_{L_{p}\left(\mathbb{R}^{d}\right)}+N^{\prime}\left(e^{-n|x|^{2} / N}+e^{-\sqrt{n}|x| / N}\right),
\end{gathered}
$$

where $N^{\prime}$ is independent of $n$. By sending $n \rightarrow \infty$ and taking onto account that $R \leq 1$ we come to

$$
\begin{aligned}
\mid R_{1}\left(B_{R}\right) f(x) & -R_{1}\left(B_{R}\right) f(0) \mid \leq N R^{2-d / p}\|f\|_{L_{p}\left(\mathbb{R}^{d}\right)} \\
& +N(|x| / R)^{\alpha}\|f\|_{L_{p}\left(\mathbb{R}^{d}\right) .}
\end{aligned}
$$

By applying this result to (4.2) and using Corollary 3.5, according to which $|u| \leq N\|f\|_{L_{p}\left(\mathbb{R}^{d}\right)}$, and also using (3.5), which implies $R_{1}\left(B_{R}\right) I_{B_{R}} \leq$ $N R^{d / p}$ for $R \leq 1$, we obtain

$$
\begin{gathered}
\left|R_{1} f(x)-R_{1} f(0)\right| \leq N\left(R^{2-d / p}+R^{d / p}\right)\|f\|_{L_{p}\left(\mathbb{R}^{d}\right)} \\
+N(|x| / R)^{\alpha}\|f\|_{L_{p}\left(\mathbb{R}^{d}\right)}
\end{gathered}
$$


If $2-d / p \leq d / p$, we take here $R=|x|^{\beta}$, where $\alpha \beta^{-1}=2-d / p+\alpha$, and we get

$$
\left|R_{1} f(x)-R_{1} f(0)\right| \leq N|x|^{\alpha(1-\beta)}\|f\|_{L_{p}\left(\mathbb{R}^{d}\right)},
$$

provided that $|x| \leq R / 12=|x|^{\beta} / 12$, that is $|x| \leq \eta=\eta(d, \delta, p,\|b\|)$. For $|x| \geq \eta$ estimate (4.10) holds due to Theorem 3.3. However, if $2-d / p>d / p$, we find $\beta$ from $\beta(d / p+\alpha)=\alpha$ and again come to (4.10).

This proves (4.1) for $\lambda=1$ and $y=0$. Shifting the coordinates take care of arbitrary $x, y$. The theorem is proved.

To prove that $X$ is strong Feller we need the following generalization of a result of Lions [15], proved in case $b$ is bounded and $p>d$, which was generalized in [6] albeit when $b=0$ but with $p \geq d_{0}$.

Theorem 4.8. For any $p \geq d_{0}$ there are constants $N$ and $\mu>0$, depending only on $d, p, \delta$, and $\|b\|$, such that for any Borel nonnegative $f$ given on $\mathbb{R}^{d}$ and $t>0$ we have

$$
T_{t} f(0) \leq N t^{-d /(2 p)}\left\|\Phi_{t} f\right\|_{L_{p}\left(\mathbb{R}^{d}\right)},
$$

where $\Phi_{t}(x)=\exp (-\mu|x| / \sqrt{t})$.

Proof. The proof is quite similar to what is done in [15] and [6]. First fix $\varepsilon \in(0,1)$ and let $F_{\varepsilon}$ be the set of Borel $f$ such that $\varepsilon \leq f(x) \leq \varepsilon^{-1}$ for any $x$. Then introduce

$$
K_{\varepsilon}=\sup \frac{T_{1} f(0)}{\left\|\Phi_{1} f\right\|_{L_{p}\left(\mathbb{R}^{d}\right)}},
$$

where the choice of $\mu$ (in $\Phi_{1}$ ) will be specified later and the supremum is taken over all $f \in F_{\varepsilon}$ and over all diffusion processes $X$, for which Assumption 1.1 is satisfied ( $\delta$ and $\|b\|$ are fixed). Obviously, $K_{\varepsilon}<\infty$. Also observe that self-similarity arguments easily show that (4.11) holds with $N=K_{\varepsilon}$ if $f \in F_{\varepsilon}$. Shifting the origin shows that for $f \in F_{\varepsilon}, t>0$, and $x \in \mathbb{R}^{d}$

$$
T_{t} f(x) \leq K_{\varepsilon} t^{-d /(2 p)}\left(\int_{\mathbb{R}^{d}} e^{-p \mu|x-y| / \sqrt{t}} f^{p}(y) d y\right)^{1 / p} .
$$

Now, define $u(t, x)=T_{t} f(x)$ and observe that by the Markov property for $s \in(0,1)$

$$
\begin{gathered}
u(1,0)=T_{s} u(1-s, \cdot)(0)=2 \int_{0}^{1 / 2} T_{s} u(1-s, \cdot)(0) d s \\
=2 E_{0} \int_{0}^{1 / 2} u\left(1-s, x_{s}\right) d s \leq N E_{0} \int_{0}^{\infty} I_{s \leq 1 / 2} e^{-s} u\left(1-s, x_{s}\right) d s .
\end{gathered}
$$

By using Theorem 3.4 we obtain

$$
u^{d+1}(1,0) \leq N I J
$$

where

$$
I=\sup _{[0,1 / 2] \times \mathbb{R}^{d}}\left(u^{d}(1-s, x) e^{-\mu|x| d}\right)=\sup _{[1 / 2,1] \times \mathbb{R}^{d}}\left(u^{d}(t, x) e^{-\mu|x| d}\right),
$$




$$
J=\int_{\mathbb{R}^{d}} e^{-\mu|x|}\left(\int_{1 / 2}^{1} u(t, x) d t\right) d y
$$

As is easy to see

$$
J \leq e \int_{\mathbb{R}^{d}} e^{-\mu|x|} R_{1} f(x) d x,
$$

which by Theorem 3.3 yields

$$
J \leq N \int_{\mathbb{R}^{d}} e^{-\mu|x|}\left(\int_{\mathbb{R}^{d}} e^{-\mu p|x-y|} f^{p}(y) d y\right)^{1 / p} d x,
$$

where, perhaps, the second $\mu$ is different from the first one. We allow ourselves to use $\mu$ as a generic constant $>0$ depending only on $p, d, \delta$, and $\|b\|$. By Hölder's inequality

$$
J \leq N\left(\int_{\mathbb{R}^{d}} e^{-\mu|x|} d x\right)^{1 / q}\left(\int_{\mathbb{R}^{d}} g(y) f^{p}(y) d y\right)^{1 / p},
$$

where $q=p /(p-1)$ and

$$
g(y)=\int_{\mathbb{R}^{d}} e^{-\mu(|x|+|x-y|)} d x .
$$

Since $|x|+|x-y| \geq(1 / 2)(|x|+|y|)$, we have

$$
J \leq N\left(\int_{\mathbb{R}^{d}} e^{-p \mu|y|} f^{p}(y) d y\right)^{1 / p} .
$$

In what concerns $I$ observe that owing to (4.12) for $t \in[1 / 2,1]$ we have

Hence

$$
\begin{gathered}
u(t, x) e^{-\mu|x|} \leq N K_{\varepsilon}\left(\int_{\mathbb{R}^{d}} e^{-\mu(|x|+|x-y|)} f^{p}(y) d y\right)^{1 / p} \\
\leq N K_{\varepsilon}\left(\int_{\mathbb{R}^{d}} e^{-p \mu|y|} f^{p}(y) d y\right)^{1 / p}
\end{gathered}
$$

$$
I \leq N K_{\varepsilon}^{d}\left(\int_{\mathbb{R}^{d}} e^{-p \mu|y|} f^{p}(y) d y\right)^{d / p},
$$

and coming back to (4.13) we get

$$
u(1,0) \leq N K_{\varepsilon}^{d /(d+1)}\left(\int_{\mathbb{R}^{d}} e^{-p \mu|y|} f^{p}(y) d y\right)^{1 / p} .
$$

Because of the definition of $K_{\varepsilon}$ it follows that

$$
K_{\varepsilon} \leq N K_{\varepsilon}^{d /(d+1)}, \quad K_{\varepsilon} \leq N .
$$

After that it only remains to send $\varepsilon \downarrow 0$ observing that the last $N$ as well as $\mu$ depend only on $p, d, \delta$, and $\|b\|$. The theorem is proved.

Remark 4.2. Once we know that (4.11) holds for Borel nonnegative $f$, we can repeat the argument from the beginning of the above proof and conclude that for all Borel nonnegative $f, t>0$, and $x \in \mathbb{R}^{d}$,

$$
T_{t} f(x) \leq N t^{-d /(2 p)}\left(\int_{\mathbb{R}^{d}} e^{-p \mu|x-y| / \sqrt{t}} f^{p}(y) d y\right)^{1 / p} .
$$


Corollary 4.9. For $p \geq d_{0}$ such that $p>d / 2+1$ there exists a constant $N=N(p, d, \delta,\|b\|)$ such that for any $T \in(0, \infty)$ and nonnegative Borel $f(t, x)$ given on $[0, T] \times \mathbb{R}^{d}$ we have

$$
I:=E_{0} \int_{0}^{T} f\left(t, x_{t}\right) d t \leq N T^{(p-1) / p-d /(2 p)}\left\|\Phi_{T} f\right\|_{L_{p}\left([0, T] \times \mathbb{R}^{d}\right)} .
$$

Indeed,

$$
I=\int_{0}^{T} T_{t} f(t, \cdot)(0) d t \leq N \int_{0}^{T} t^{-d /(2 p)}\left(\int_{\mathbb{R}^{d}} \Phi_{T}^{p}(y) f^{p}(t, y) d y\right)^{1 / p} d t
$$

and it only remains to use Hölder's inequality.

Remark 4.3. Observe that the usual parabolic Aleksandrov estimate gives (4.16) with $p \geq d+1$. We were able to reduce $p$ because $a$ is independent of $t$. Also note that in [8] there is an example showing that (4.16) and (4.15) generally (when $a$ is independent of $t$ ) fail to hold for any fixed $p<d$ if $\delta$ can be chosen small enough. In that regard see also the example in [6], that appeared a few years later than [8].

The following is deduced from (4.15) in the same way as (3.6) in Corollary 3.5 is derived from Theorem 3.4.

Corollary 4.10. For $q \geq p \geq d_{0}$ there exists a constant $N=N(p, q, d, \delta,\|b\|)$ such that for any $t>0$ and nonnegative Borel $f$

$$
\left\|T_{t} f\right\|_{L_{q}\left(\mathbb{R}^{d}\right)} \leq N t^{(d / 2)(1 / q-1 / p)}\|f\|_{L_{p}\left(\mathbb{R}^{d}\right)} .
$$

Remark 4.4. The fact that the semigroup $T_{t}$ is bounded in $L_{p}$, which follows from Corollary 4.10 with $p=q$, should not look very surprising and follows by self-similarity from the boundedness of $T_{1}$ (which, however, is not trivial).

Corollary 4.11. For $p \geq d_{0}$ and $f \in L_{p}\left(\mathbb{R}^{d}\right)$

$$
\lim _{t \downarrow 0}\left\|T_{t} f-f\right\|_{L_{p}\left(\mathbb{R}^{d}\right)}=0 .
$$

Indeed, in light of Corollary 4.10 (with $q=p$ ) we may concentrate on $f \in C_{0}^{\infty}\left(\mathbb{R}^{d}\right)$. In that case by Itô's formula

$$
T_{t} f-f=\int_{0}^{t} T_{s} L f d s
$$

where $L f \in L_{d_{0}}\left(\mathbb{R}^{d}\right)$. Hence, by Corollary 4.10

$$
\left\|T_{t} f-f\right\|_{L_{p}\left(\mathbb{R}^{d}\right)} \leq \int_{0}^{t}\left\|T_{s} L f\right\|_{L_{p}\left(\mathbb{R}^{d}\right)} d s \leq \int_{0}^{t} s^{(d / 2)\left(1 / p-1 / d_{0}\right)} d s\|L f\|_{L_{d_{0}}\left(\mathbb{R}^{d}\right)}
$$

and the last integral tends to zero as $t \downarrow 0$ since $d_{0}>d / 2$.

Theorem 4.12. The process $X$ is strong Feller in the sense that for any bounded Borel $f$ given on $\mathbb{R}^{d}$ the function $T_{t} f(x)$ is continuous on $(0, \infty) \times$ $\mathbb{R}^{d}$. 
Proof. In light of Theorem 4.8 we may concentrate on smooth compactly supported $f$ 's. In that case we are going to prove that $T_{t} f(x)$ is continuous in $[0, \infty) \times \mathbb{R}^{d}$. Observe that for $t \geq s \geq 0$ we have

$$
\begin{gathered}
\left|T_{t} f(x)-T_{s} f(x)\right|=\left|T_{s}\left(T_{t-s} f-f\right)(x)\right|=\left|E_{x} E_{x_{s}}\left[f\left(x_{t-s}\right)-f\left(x_{0}\right)\right]\right| \\
\leq \sup |\nabla f| \sup _{y} E_{y}\left|x_{t-s}-x_{0}\right| \leq N \sup |\nabla f| \sqrt{t-s},
\end{gathered}
$$

where the last inequality follows from Theorem 3.1. We see that $T_{t} f(x)$ is continuous in $[0, \infty)$ uniformly with respect to $x$.

Next, for our $f$ Theorem 3.1 easily implies that $\lambda R_{\lambda} f \rightarrow f$ as $\lambda \rightarrow \infty$ uniformly with respect to $x$. After that the continuity of $T_{t} f(x)$ with respect to $x$ follows from the fact that

$$
T_{t} \lambda R_{\lambda} f=\lambda R_{\lambda} T_{t} f
$$

where the right-hand sides are continuous in $x$ due to Theorem 4.1 and the left-hand sides converge uniformly in $x$ to $T_{t} f(x)$. The theorem is proved.

We finish the section by a version of parabolic Aleksandrov estimates.

Theorem 4.13. Let $D$ be a bounded domain in $\mathbb{R}^{d}, p \geq d_{0}, p>d / 2+1$, $T \in(0, \infty)$ and let $u \in W_{p, \text { loc }}^{1,2}((0, T) \times D) \cap C([0, T] \times \bar{D})$. Then there is a constant $N$, depending only on $d, \delta,\|b\|$, and $T$, such that in $[0, T] \times D$ we have

$$
u \leq N\left\|\left(\partial_{t} u+L u\right)_{-}\right\|_{L_{p}((0, T) \times D)}+\sup _{(\partial((0, T) \times D)) \backslash(\{0\} \times D)} u .
$$

Proof. In the same way as in the proof of Theorem 3.1 of [12] we convince ourselves that we may assume that $u \in W_{p}^{1,2}((0, T) \times D), D$ is smooth, and $b$ is bounded. In that case it suffices to prove (4.18) for $u \in C^{1,2}([0, T] \times \bar{D})$. For such $u$, by Itô's formula $P_{0}$-(a.s.) for all $t \leq T$

$u\left(t \wedge \tau, x_{t \wedge \tau}\right)=u(0,0)+\int_{0}^{t \wedge \tau}\left(\partial_{t} u+L u\right)\left(s, x_{s}\right) d s+\int_{0}^{t \wedge \tau} D_{i} u\left(s, x_{s}\right) \sigma^{i k}\left(x_{s}\right) d w_{s}^{k}$

and the stochastic integral is a martingale, where $\tau$ is the first exit time of $\left(t, x_{t}\right)$ from $[0, T) \times D$. By setting $t=T$ and taking expectations we get

$$
\begin{gathered}
u(0,0)=-E_{0} \int_{0}^{\tau}\left(\partial_{t} u+L u\right)\left(t, x_{t}\right) d t+E_{0} u\left(\tau, x_{\tau}\right) \\
\leq E_{0} \int_{0}^{\tau}\left(\partial_{t} u+L u\right)_{-}\left(t, x_{t}\right) d t+\sup _{(\partial((0, T) \times D)) \backslash(\{0\} \times D)} u .
\end{gathered}
$$

After that it only remains to use Corollary 4.9. The theorem is proved. 


\section{Estimating time spent in SPaCe-time sets of Small measure}

Here we present extensions to the case that $b \in L_{d}$ of probabilistic versions of some PDE results found in [14], [17], [11]. Recall the notation introduced before Theorem 3.8 and also introduce

$$
\begin{aligned}
& C_{T, R}^{o}=(0, T) \times B_{R}, \quad C_{T, R}^{o}(t, x)=(t, x)+C_{T, R}^{o}, \quad C_{R}^{o}(t, x)=C_{R^{2}, R}^{o}(t, x), \\
& C_{R}^{o}=C_{R}^{o}(0,0) \text {. Fix } \\
& q, \eta, \kappa \in(0,1) .
\end{aligned}
$$

For cylinders $Q=C_{\rho}^{o}(t, x)$ define

$$
\begin{gathered}
Q^{\prime}=(t, x)-C_{\eta^{-1} \rho^{2}, \rho}^{o}, \quad Q^{\prime \prime}=\left(t-\eta^{-1} \rho^{2}, x\right)+C_{\eta^{-1} \rho^{2} \kappa^{2}, \rho \kappa}^{o}, \\
Q_{+}^{\prime}=Q \cup Q^{\prime} \cup\left(\{t\} \times B_{\rho}(x)\right) .
\end{gathered}
$$

Imagine that the $t$-axis is pointed up vertically. Then $Q^{\prime}$ is adjacent to $Q$ from below, the two cylinders have a common base, and along the $t$-axis $Q^{\prime}$ is $\eta^{-1}$ times longer than $Q$. The cylinder $Q^{\prime \prime}$ is obtained by contracting $Q^{\prime}$ to the center of its lower base with the contraction factor $\kappa^{-2}$ for the $t$-axis and $\kappa^{-1}$ for the spatial axes.

Remark 5.1. If $Q=C_{\rho}^{o}(t, x)$, then the shortest distance between $Q$ and $Q^{\prime \prime}$ along the $t$ axis is

$$
\eta^{-1} \rho^{2}-\eta^{-1} \rho^{2} \kappa^{2}=\eta^{-1} \rho^{2}\left(1-\kappa^{2}\right),
$$

which is bigger than $2 \rho^{2}$ if

$$
\kappa^{2} \leq 1-2 \eta
$$

Let $\Gamma$ be a measurable subset of $C_{1}$ and introduce $\mathcal{B}=\mathcal{B}(\Gamma, q)$ as the family of open cylinders $Q$ of type $C_{\rho}^{o}\left(t_{0}, x_{0}\right)$ such that

$$
Q \subset C_{1} \text { and }|Q \cap \Gamma| \geq q|Q| \text {. }
$$

Finally, define

$$
\Gamma^{\prime \prime}=\bigcup_{Q \in \mathcal{B}} Q^{\prime \prime}, \quad \Gamma_{\varepsilon}^{\prime \prime}=\bigcup_{Q \in \mathcal{B}:|Q| \geq \varepsilon} Q^{\prime \prime} .
$$

Observe that for $Q \in \mathcal{B}$ the set $Q^{\prime \prime}$ is open. Hence, $\Gamma^{\prime \prime}$ is open and measurable.

Lemma 5.1. If $|\Gamma| \leq q\left|C_{1}\right|$, then

$$
\left|\Gamma^{\prime \prime}\right| \geq\left(1-\frac{1-q}{3^{d+1}}\right)^{-1}(1+\eta)^{-1} \kappa^{d+2}|\Gamma|
$$

and there exists $\theta=\theta(d, q, \eta, \kappa)>1$ such that for any sufficiently small $\varepsilon>0$ there exists a closed $\Gamma_{\varepsilon} \subset \Gamma_{\varepsilon}^{\prime \prime}$ such that

$$
\left|\Gamma_{\varepsilon}\right| \geq \theta|\Gamma| \text {. }
$$

The first assertion of the lemma originated in [14], [17], is presented, for instance as Lemma 9.3.6 in [11]. The second one is proved in the same way as the second assertion of Lemma 4.7 of [13]. 
Lemma 5.2. Let $\kappa \in(0,1)$. Then there is a constant $q_{0}=q_{0}(\kappa, d, \delta,\|b\|) \in$ $(0,1)$ such that for any $R \in(0, \infty)$, Borel set $\Gamma \subset C_{R}$ satisfying $|\Gamma| \geq q_{0}\left|C_{R}\right|$, and $x \in B_{\kappa R}$ we have

$$
E_{x} \int_{0}^{\tau_{R} \wedge R^{2}} I_{\Gamma}\left(t, x_{t}\right) d t \geq \mu_{0} R^{2},
$$

where $\mu_{0}=\mu_{0}(d, \delta,\|b\|, \kappa) \in(0,1)$.

Proof. As usual we let $R=1$. Then observe that by Lemma 2.13 of [12] we have $E_{x}\left(\tau_{1} \wedge 1\right) \geq \nu=\nu(d, \delta,\|b\|, \kappa)>0$. By using Corollary 4.9 we get that

$$
\begin{aligned}
& E_{x}\left(\tau_{1} \wedge 1\right)-E_{x} \int_{0}^{\tau_{1} \wedge 1} I_{\Gamma}\left(t, x_{t}\right) d t=E_{x} \int_{0}^{\tau_{1} \wedge 1} I_{C_{1} \backslash \Gamma}\left(t, x_{t}\right) d t \\
\leq & N\left(\left|C_{1}\right|-|\Gamma|\right)^{1 / d_{0}} \leq N\left(1-q_{0}\right)^{1 / d_{0}} \leq N\left(1-q_{0}\right)^{1 / d_{0}} E_{x}\left(\tau_{1} \wedge 1\right),
\end{aligned}
$$

where the constants $N$ depend only on $\kappa, d, \delta$, and $\|b\|$. We see how to choose $q_{0}$ to satisfy (5.4) with a $\mu_{0}=\mu_{0}(d, \delta,\|b\|, \kappa) \in(0,1)$. The lemma is proved.

In Lemma 5.3 by $q_{0}$ we mean the one from Lemma 5.2.

Lemma 5.3. Take $Q=C_{\rho}^{o}(s, y)$, use the notation $Q^{\prime}, Q^{\prime \prime}, Q_{+}^{\prime}$ introduced above, assume (5.2), and suppose that Borel $\Gamma \subset Q$ is such that $|\Gamma| \geq q_{0}|Q|$. Then there is a constant $\nu_{0}>0$, depending only on $\eta, \kappa, d, \delta,\|b\|$, such that for any $\left(t_{0}, x_{0}\right) \in Q^{\prime \prime}$

$$
E_{x_{0}} \int_{0}^{\tau} I_{\Gamma}\left(t, x_{t}\right) d t \geq \nu_{0} E_{x_{0}} \tau
$$

where $\tau$ is the first exit time of $\left(t_{0}+t, x_{t}\right)$ from $Q_{+}^{\prime}$.

Proof. Thanks to (5.2) and Remark 5.1 we have $s-t_{0} \in\left(2 \rho^{2}, \eta^{-1} \rho^{2}\right)$. Also $\left|y-x_{0}\right|<\kappa \rho$. It follows by Theorem 3.9 that

$$
P_{x_{0}}\left(\sup _{r \in\left[0, s-t_{0}\right]}\left|x_{r}-y\right|<\rho,\left|x_{s-t_{0}}-y\right|<\kappa \rho\right) \geq \nu,
$$

where $\nu=\nu(\kappa, \eta, d, \delta,\|b\|)>0$.

Next. for $\gamma$ defined as the first exit time of $\left(t_{0}+t, x_{t}\right)$ from $Q^{\prime}$ in light of Lemma 5.2 we have

$$
\begin{gathered}
E_{x_{0}} \int_{0}^{\tau} I_{\Gamma}\left(t, x_{t}\right) d t=E_{x_{0}} I_{\gamma>s-t_{0}} \int_{\gamma}^{\tau} I_{\Gamma}\left(t, x_{t}\right) d t \\
\geq E_{x_{0}} I_{\gamma>s-t_{0},\left|x_{s-t_{0}}-y\right|<\kappa \rho} E_{x_{s-t_{0}}} \int_{0}^{\tau} I_{\Gamma}\left(t, x_{t}\right) d t \\
\geq \mu_{0} \rho^{2} P_{x_{0}}\left(\sup _{r \in\left[0, s-t_{0}\right]}\left|x_{r}-y\right|<\rho,\left|x_{s-t_{0}}-y\right|<\kappa \rho\right) \geq \mu_{0} \nu \rho^{2} .
\end{gathered}
$$

On the other hand, the height of $Q_{+}^{\prime}$ is $\left(1+\eta^{-1}\right) \rho^{2}$, so that $\left(t_{0}+t, x_{t}\right)$ cannot spend in $Q_{+}^{\prime}$ more time than $\left(1+\eta^{-1}\right) \rho^{2}$. This proves the lemma. 
Theorem 5.4. For any $\kappa \in(0,1)$ there exists $\gamma \in(0,1)$ and $N$, depending only on $\kappa, d, \delta,\|b\|$, such that for any $R \in(0, \infty), q \in(0,1)$, Borel $\Gamma \subset$ $C_{R}\left(R^{2}, 0\right)$ satisfying $|\Gamma| \geq q\left|C_{R}\left(R^{2}, 0\right)\right|$, and $x \in B_{\kappa R}$ we have

$$
G_{R}(\Gamma, x):=E_{x} \int_{0}^{\tau_{R} \wedge\left(2 R^{2}\right)} I_{\Gamma}\left(t, x_{t}\right) d t \geq N^{-1} q^{1 / \gamma} R^{2} .
$$

Proof. Self-similar transformations allow us to assume that $R=1$ and write $G(\Gamma, x)$ instead of $G_{1}(\Gamma, x)$. Then find and fix $q_{0}, \eta, \kappa \in(0,1)$, depending only on $d, \delta,\|b\|$, such that (5.2) holds, $\theta$ from Lemma 5.1 is strictly bigger than 1, and (5.4) holds whenever $|\Gamma| \geq q_{0}\left|C_{R}\right|$. Clearly we can find such $\kappa \in(0,1)$ which is larger than the one in the statement of the theorem.

It is convenient to introduce a function $\mu(q)$ as the infimum of the lefthand sides of (5.6) (with $R=1$ ) over all Borel $\Gamma \subset C_{1}(1,0)$ satisfying $|\Gamma| \geq q\left|C_{1}(1,0)\right|$ and over all $x \in B_{\kappa}$. Observe that a combination of Lemma 5.2 and Theorem 3.9, as in the proof of Lemma 5.3, leads to the conclusion that there exists $q_{0}, \mu_{0} \in(0,1)$, depending only on $\eta, \kappa, d, \delta,\|b\|$, such that

for $q \in\left[q_{0}, 1\right]$.

$$
\mu(q) \geq \mu_{0}
$$

We will be comparing $\mu\left(q^{\prime}\right)$ and $\mu\left(q^{\prime \prime}\right)$ for $0<q^{\prime}<q^{\prime \prime}<1$ such that

$$
(1+\theta) q^{\prime} \geq 2 q^{\prime \prime}
$$

We take a Borel $\Gamma \subset C_{1}(1,0)$ satisfying $|\Gamma| \geq q^{\prime}\left|C_{1}(1,0)\right|$ and in the construction before Lemma 5.1 we replace $C_{1}$ by $C_{1}(1,0)$, keep all other notation, and from the chosen $\Gamma, \kappa, \eta$, and $q_{0}$ (not $q^{\prime}$ ) we build up the sets $\Gamma_{\varepsilon}$ and take $\varepsilon$ so small that (5.3) holds. There are two cases:

(i) $\left|\Gamma_{\varepsilon} \backslash C_{1}(1,0)\right| \leq\left(q^{\prime \prime}-q^{\prime}\right)\left|C_{1}\right|$

(ii) $\left|\Gamma_{\varepsilon} \backslash C_{1}(1,0)\right|>\left(q^{\prime \prime}-q^{\prime}\right)\left|C_{1}\right|$.

Case ( $i$ ). Our goal is to show that

$$
G(\Gamma, x) \geq \min \left(\mu_{0}, \nu_{0} \mu\left(q^{\prime \prime}\right)\right), \quad|x| \leq \kappa,
$$

where $\nu_{0}$ depends only on $\kappa, \eta, d, \delta,\|b\|$.

Observe that, if $|\Gamma| \geq q_{0}\left|C_{1}\right|$, by definition $G(\Gamma, x) \geq \mu\left(q_{0}\right) \geq \mu_{0}$ for $|x| \leq R$. Hence, we may assume that

$$
|\Gamma|<q_{0}\left|C_{1}\right|
$$

In that case define

$$
\hat{\Gamma}_{\varepsilon}=\Gamma_{\varepsilon} \cap C_{1}^{o}(1,0) .
$$

Notice that by definition and Lemma 5.1

$$
q^{\prime}\left|C_{1}\right| \leq|\Gamma| \leq \theta^{-1}\left|\Gamma_{\varepsilon}\right|
$$

Moreover, by assumption

$$
\left|\Gamma_{\varepsilon}\right|=\left|\Gamma_{\varepsilon} \backslash C_{1}(1,0)\right|+\left|\hat{\Gamma}_{\varepsilon}\right| \leq\left(q^{\prime \prime}-q^{\prime}\right)\left|C_{1}\right|+\left|\hat{\Gamma}_{\varepsilon}\right| .
$$

Due to (5.7), it follows that

$$
\left|\hat{\Gamma}_{\varepsilon}\right| \geq q^{\prime \prime}\left|C_{1}\right|,
$$


so that

$$
G\left(\hat{\Gamma}_{\varepsilon}, x\right) \geq \mu\left(q^{\prime \prime}\right), \quad|x| \leq \kappa .
$$

We now estimate $G(\Gamma, x)$ from below by means of $G\left(\hat{\Gamma}_{\varepsilon}, x\right)$ using Lemma 5.3. Since $\Gamma_{\varepsilon} \subset \Gamma_{\varepsilon}^{\prime \prime}$, the closed set $\Gamma_{\varepsilon}$ is covered by the family $\left\{Q^{\prime \prime}: Q \in\right.$ $\mathcal{B},|Q| \geq \varepsilon\}$. Then there is finitely many $Q(1), \ldots, Q(n) \in \mathcal{B}$ such that $|Q(i)| \geq \varepsilon, i=1, \ldots, n$, and

$$
\Gamma_{\varepsilon} \subset \bigcup_{i=1}^{n} Q^{\prime \prime}(i)=: \Pi_{\varepsilon} .
$$

Then for $(t, x) \in \Pi_{\varepsilon}$ define $i(t, x)$ as the first $i \in\{1, \ldots, n\}$ for which $(t, x) \in Q^{\prime \prime}(i)$. Also set $Q_{+}^{\prime}(0)=C_{2,1}$ and $i(t, x)=0$ if $(t, x) \in \partial C_{2,1}$. Now define recursively $\gamma^{0}=0, \tau^{1}$ as the first time after $\gamma^{0}$ when $\left(t, x_{t}\right)$ exits from $C_{2,1} \backslash \Gamma_{\varepsilon}, \gamma^{1}$ as the first time after $\tau^{1}$ when $\left(t, x_{t}\right)$ exits from $Q_{+}^{\prime}\left(i\left(\tau^{1}, x_{\tau^{1}}\right)\right)$, and generally, for $k=2,3, \ldots$ define $\tau^{k}$ as the first time after $\gamma^{k-1}$ when $\left(t, x_{t}\right)$ exits from $C_{2,1} \backslash \Gamma_{\varepsilon}, \gamma^{k}$ as the first time after $\tau^{k}$ when $\left(t, x_{t}\right)$ exits from $Q_{+}^{\prime}\left(i\left(\tau^{k}, x_{\tau^{k}}\right)\right)$. It is easy to check that so defined $\tau^{k}$ and $\gamma^{k}$ are stopping times and, since $|Q(i)| \geq \varepsilon$ and the trajectories of $\left(t, x_{t}\right)$ are continuous, $\tau^{k} \uparrow \tau_{1} \wedge 2$ as $k \rightarrow \infty$. Furthermore, (a.s.) all the $\tau^{k}$ 's equal $\tau_{1} \wedge 2$ for all large $k$.

For a domain $Q \subset \mathbb{R}^{d+1}$ we denote by $\gamma(s, Q)$ the first exit time of $\left(s+t, x_{t}\right)$ from $Q$ and by the strong Markov property obtain

$$
\begin{gathered}
G(\Gamma, x) \geq \sum_{k=1}^{\infty} E_{x} \int_{\tau^{k}}^{\gamma^{k}} I_{\Gamma}\left(t, x_{t}\right) d t \\
=\left.\sum_{k=1}^{\infty} E_{x} E_{x_{\tau^{k}}} \int_{0}^{\gamma\left(s, Q_{+}^{\prime}(i)\right)} I_{\Gamma}\left(s+t, x_{t}\right) d t\right|_{i=i\left(\tau^{k}, x_{\tau^{k}}\right), s=\tau^{k}} .
\end{gathered}
$$

We estimate the interior expectation from below by Lemma 5.3 and get

$$
\begin{aligned}
& G(\Gamma, x) \geq\left.\nu_{0} \sum_{k=1}^{\infty} E_{x} E_{x_{\tau^{k}}} \int_{0}^{\gamma\left(s, Q_{+}^{\prime}(i)\right)} I_{\Pi_{\varepsilon}}\left(s+t, x_{t}\right) d t\right|_{s=\tau^{k}, i=i\left(\tau^{k}, x_{\tau^{k}}\right)} \\
& \geq\left.\nu_{0} \sum_{k=1}^{\infty} E_{x} E_{x_{\tau^{k}}} \int_{0}^{\gamma\left(s, Q_{+}^{\prime}(i)\right)} I_{\Gamma_{\varepsilon}}\left(s+t, x_{t}\right) d t\right|_{s=\tau^{k}, i=i\left(\tau^{k}, x_{\tau^{k}}\right)} \\
& =\nu_{0} \sum_{k=1}^{\infty} E_{x} \int_{\tau^{k}}^{\gamma^{k}} I_{\Gamma_{\varepsilon}}\left(t, x_{t}\right) d t=\nu_{0} G\left(\Gamma_{\varepsilon}, x\right) \geq \nu_{0} G\left(\hat{\Gamma}_{\varepsilon}, x\right) \geq \nu_{0} \mu\left(q^{\prime \prime}\right) .
\end{aligned}
$$

This proves (5.8).

Case (ii). Here the goal is to prove that

$$
G(\Gamma, x) \geq \mu_{0} \nu \eta^{n}\left(q^{\prime \prime}-q^{\prime}\right)^{n}, \quad|x| \leq \kappa,
$$

where $\nu>0$ and $n \geq 1$ depend only on $d, \delta,\|b\|, \eta$, and $\kappa$. 
First we claim that for some $(t, x) \in \Gamma_{\varepsilon}$ it holds that $t<q^{\prime}-q^{\prime \prime}+1$. Indeed, otherwise $\Gamma_{\varepsilon} \backslash C_{1}(1,0) \subset C_{q^{\prime \prime}-q^{\prime}, 1}\left(q^{\prime}-q^{\prime \prime}+1,0\right)$ and $\left|\Gamma_{\varepsilon} \backslash C_{1}(1,0)\right| \leq$ $\left(q^{\prime \prime}-q^{\prime}\right)\left|C_{1}\right|$. It follows that there is a cylinder

$$
Q=C_{\rho}^{o}(s, y) \in \mathcal{B}
$$

such that $Q^{\prime}$ contains points in the half-space $t<q^{\prime}-q^{\prime \prime}+1$. Since $q^{\prime}<q^{\prime \prime}$, we have $q^{\prime}-q^{\prime \prime}+1<1$, and since $Q^{\prime}$ is adjacent to $Q \subset C_{1}(1,0)$, this implies that the height of $Q^{\prime}$ is at least $q^{\prime \prime}-q^{\prime}$, that is,

$$
\rho^{2} \eta^{-1} \geq q^{\prime \prime}-q^{\prime}, \quad \rho^{2} \geq \eta\left(q^{\prime \prime}-q^{\prime}\right) .
$$

On the other hand, $Q \subset C_{1}(1,0), s>1$, and $\rho<1$.

Moreover, by construction, $|\Gamma \cap Q| \geq q_{0}|Q|$ and by Lemma 5.2

$$
E_{x} \int_{0}^{\tau} I_{\Gamma}\left(s+t, x_{t}\right) d t \geq \mu_{0} \rho^{2} \geq \mu_{0} \eta\left(q^{\prime \prime}-q^{\prime}\right)
$$

if $|x-y| \leq \kappa \rho$, where $\tau$ is the first exit time of $\left(s+t, x_{t}\right)$ from $C_{\rho}(s, y)$. Now by Theorem 3.9 for $x \in B_{\kappa}$

$$
\begin{aligned}
& E_{x} \int_{0}^{\tau_{2,1}} I_{\Gamma}\left(t, x_{t}\right) d t \geq E_{x} I_{\tau_{1}>s,\left|x_{s}-y\right| \leq \kappa \rho} E_{x_{s}} \int_{0}^{\tau} I_{\Gamma}\left(s+t, x_{t}\right) d t \\
& \geq \mu_{0} \eta\left(q^{\prime \prime}-q^{\prime}\right) P_{x}\left(\tau_{1}>s,\left|x_{s}-y\right| \leq \kappa \rho\right) \geq N^{-1} \rho^{\nu} \mu_{0} \eta\left(q^{\prime \prime}-q^{\prime}\right) .
\end{aligned}
$$

This proves (5.9).

By combining the two cases (i) and (ii) we conclude that

$$
G(\Gamma, x) \geq \min \left(\mu_{0}, \nu_{0} \mu\left(q^{\prime \prime}\right), \mu_{0} \nu \eta^{n}\left(q^{\prime \prime}-q^{\prime}\right)^{n}\right), \quad|x| \leq \kappa,
$$

and the arbitrariness of $\Gamma$ allows us to conclude that

$$
\mu\left(q^{\prime}\right) \geq \min \left(\mu_{0}, \nu_{0} \mu\left(q^{\prime \prime}\right), \mu_{0} \nu \eta^{n}\left(q^{\prime \prime}-q^{\prime}\right)^{n}\right),
$$

whenever (5.7) holds. Observe that (5.11) is identical to (9.3.10) of [11] and by literally repeating what is in [11], just replacing $\xi$ there with our $\theta$, we come to (5.6). The theorem is proved.

The following three results are derived from Theorem 5.4 in the same way as similar results are derived from Theorem 4.1 of [13].

Corollary 5.5. For any $\kappa \in(0,1)$ there exists $N=N(d, \delta,\|b\|, \kappa)$ such that, for any $R \in(0, \infty), x \in B_{\kappa R}$, and closed set $\Gamma \subset C_{R}\left(R^{2}, 0\right)$, the probability that the process $\left(t, x_{t}\right)$ with $x_{0}=x$ reaches $\Gamma$ before exiting from $C_{2 R^{2}, R}$ is greater than or equal to $N^{-1}\left(|\Gamma| /\left|C_{R}\right|\right)^{\mu-1 / d_{0}}$ :

$$
P_{x}\left(\tau_{\Gamma}<\tau_{2 R^{2}, R}\right) \geq N^{-1}\left(|\Gamma| /\left|C_{R}\right|\right)^{\mu-1 / d_{0}},
$$

where $\tau_{\Gamma}$ is the first time $\left(t, x_{t}\right)$ hits $\Gamma, \tau_{2 R^{2}, R}$ is the first exit time of $\left(t, x_{t}\right)$ from $C_{2 R^{2}, R}, \mu=1 / \gamma$, and $\gamma$ is taken from Theorem 5.4 .

Corollary 5.6. For any Borel nonnegative $f$ vanishing outside $C_{R}\left(R^{2}, 0\right)$ and $x \in B_{\kappa R}$

$$
\int_{C_{R}\left(R^{2}, 0\right)} f^{1 /(2 \mu)}(t, y) d y d t \leq N R^{d-1 / \mu}\left(E_{x} \int_{0}^{\tau_{R^{2}, R}} f\left(t, x_{t}\right) d t\right)^{1 /(2 \mu)},
$$


where $N=N(d, \delta,\|b\|, \kappa)$.

Theorem 5.7. Let $p \in\left[d_{0}, \infty\right), u \in W_{p, \mathrm{loc}}^{1,2}\left(C_{2,1}\right) \cap C\left(\bar{C}_{2,1}\right)$, and $c \in L_{d_{0}}\left(C_{2,1}\right)$ $c \geq 0$. Then

$$
\begin{gathered}
\left(\int_{C_{1,1}(1,0)}\left|D^{2} u\right|^{1 /(2 \mu)} d x d t\right)^{2 \mu} \leq N \sup _{\partial^{\prime} C_{2,1}}|u| \\
+N\left(\int_{C_{2,1}}\left|\partial_{t} u+L u-c u\right|^{p} d x d t\right)^{1 / p}
\end{gathered}
$$

where $\partial_{t}=\partial / \partial t, \partial^{\prime} C_{2,1}=\partial C_{2,1} \backslash\left(\{0\} \times B_{1}\right), \mu$ is taken from Corollary 5.5 and $N$ depends only on $d, \delta,\|b\|, p$, and $\|c\|_{L_{d_{0}}\left(C_{2,1}\right)}$.

It is worth emphasizing that in (5.13) the restriction on $p$ is $p \geq d_{0}$ and $d_{0}<d$. If $a$ depended on $t, p$ would be $>d$.

Theorem 5.7 is similar to Theorem 9.4.1 of [11] and in the same way as Theorem 9.4.9 of [11] is derived from it (by using a simple trick) one derives from Theorem 5.7 the following.

Theorem 5.8. Let $p \in\left[d_{0}, \infty\right), u \in W_{p, \text { loc }}^{1,2}\left(C_{1}\right) \cap C\left(\bar{C}_{1}\right)$, and $c \in L_{d_{0}}\left(C_{1}\right)$, $c \geq 0$. Then

$$
\left(\int_{C_{1}}\left|D^{2} u\right|^{1 /(2 \mu)} d x d t\right)^{2 \mu} \leq N\left(\int_{C_{1}}\left|\partial_{t} u+L u-c u\right|^{p} d x d t\right)^{1 / p}+N \sup _{\partial^{\prime} C_{1}}|u|,
$$

where $\partial^{\prime} C_{1}=\partial C_{1} \backslash\left(\{0\} \times B_{1}\right)$, $\mu$ is taken from Corollary 5.5 and $N$ depends only on $d, \delta,\|b\|, p$, and $\|c\|_{L_{d_{0}}\left(C_{1}\right)}$.

In the next section we will need the following.

Theorem 5.9. Let $\kappa, \eta, \zeta, q \in(0,1), R \in(0, \infty), T \in\left[\eta R^{2}, \eta^{-1} R^{2}\right]$, and closed $\Gamma \subset C_{T, R}$ be such that $\left|\Gamma \cap C_{\zeta T, R}((1-\zeta) T, 0)\right| \geq q\left|C_{\zeta T, R}\right|$. Then there exists $p_{0}=p_{0}(\kappa, \eta, \zeta, q, d, \delta,\|b\|)>0$ such that, for $\left(t_{0}, x_{0}\right) \in C_{(1-\zeta) T, \kappa R}$,

$$
P_{x_{0}}\left(\tau_{\Gamma}<\tau_{T, R}\right) \geq p_{0},
$$

where $\tau_{\Gamma}$ is the first time $\left(t_{0}+t, x_{t}\right)$ hits $\Gamma$ and $\tau_{T, R}$ is its first exit time from $C_{T, R}$.

Proof. As usual assume that $R=1$. Then observe that one can choose $\rho>0$ depending only on $d, \eta, \zeta$, and $q$ and one can find $\left(t^{0}, x^{0}\right) \in C_{T, 1}$ with $t^{0} \geq \rho^{2}+(1-\zeta) T$ such that $C_{\rho}\left(t^{0}+\rho^{2}, x^{0}\right) \subset C_{T, 1}$ and $\left|\Gamma \cap C_{\rho}\left(t^{0}+\rho^{2}, x^{0}\right)\right| \geq$ $\bar{q}\left|C_{\rho}\right|$, where $\bar{q}>0$ depends only on $d, \eta, \zeta$, and $q$. Then by Corollary 5.5, for $x \in \kappa B_{\rho}\left(x^{0}\right)$ the $P_{x}$-probability that the process $\left(t^{0}+t, x_{t}\right)$ will hit $\Gamma$ before exiting from $C_{2 \rho^{2}, \rho}\left(t^{0}, x^{0}\right)$ is estimated from below by a strictly positive constant depending only on $\kappa, \bar{q}, d, \delta,\|b\|$. After that it only remains to invoke Theorem 3.9 recalling that $t^{0} \geq \rho^{2}+(1-\zeta) T$. The theorem is proved. 


\section{Harnack inequality, Hölder Continuity of $X$-CAloric FUNCTIONS, AND SOME OTHER RESULTS}

Safonov, [18], considered the case of the coefficients of $L$ so regular that $X$-harmonic functions are sufficiently smooth and gave the estimate of the Hölder norm of $X$-harmonic functions and the estimate of the Harnack constant for them independent of the imposed regularity of $L$ in terms of only $d, \delta,\|b\|$. We emphasize that $\|b\|$ is a bound of the $L_{d}$-norm of $b$. Our case is not covered by [18], since the origin of our $X$ is unknown and it is unknown if and in which sense it can be approximated by processes with regular coefficients. At the same time some arguments here are quite close to those in [18] as well as to those in [14], [17], [11].

Definition 6.1. If $Q$ is a set in $\mathbb{R}^{d+1}=\left\{(t, x): t \in \mathbb{R}, x \in \mathbb{R}^{d}\right\}$ and $u$ is a bounded Borel function on $Q$, we call it caloric (relative to the process $X)$ if for any $(s, y)$ and $T, R \in(0, \infty)$ such that $\bar{C}_{T, R}(s, y) \subset Q$ and any $\left(t_{0}, x_{0}\right) \in C:=C_{T, R}(s, y)$ we have

$$
u\left(t_{0}, x_{0}\right)=E_{x_{0}} u\left(t_{0}+\tau_{C}, x_{\tau_{C}}\right),
$$

where $\tau_{C}$ is the first exit time of $\left(t_{0}+t, x_{t}\right)$ from $C$.

If $D$ is a set in $\mathbb{R}^{d}$ and $u$ is a bounded Borel function on $D$, we call it harmonic (relative to the process $X$ ) if for any $y$ and $R \in(0, \infty)$ such that $\bar{B}_{R}(y) \subset D$ and any $x \in B_{R}(y)$ we have

$$
u(x)=E_{x} u\left(x_{\tau_{R}(y)}\right),
$$

where $\tau_{R}(y)$ is the first exit time of $x_{t}$ from $B_{R}(y)$.

Remark 6.1. If $u$ is harmonic in $D$ and $\bar{B}_{R}(y) \subset D$ and $x \in B_{R}(y)$, then by using the Markov property of $X$ we find

$$
\begin{gathered}
u(x)=E_{x} E\left(u\left(x_{\tau_{R}(y)}\right) \mid \mathcal{N}_{T}\right)=E_{x} I_{\tau_{R}(y) \leq T} u\left(x_{\tau_{R}}\right)+E_{x} I_{\tau_{R}(y)>T} E_{x_{T}} u\left(x_{\tau_{R}(y)}\right) \\
=E_{x} I_{\tau_{R}(y) \leq T} u\left(x_{\tau_{R}(y)}\right)+E_{x} I_{\tau_{R}(y)>T} u\left(x_{T}\right)=E_{x} u\left(x_{\tau_{R}(y) \wedge T}\right)
\end{gathered}
$$

which implies that $u$ is a caloric function in $\mathbb{R} \times D$. Also if $u$ is caloric in $Q$, $\bar{C}_{T, R}(s, y) \subset Q$ and $\left(t_{0}, x_{0}\right) \in C:=C_{T, R}(s, y)$, then by the strong Markov property for any stopping time $\tau \leq \tau_{C}$ we have

$$
u\left(t_{0}, x_{0}\right)=E_{x_{0}} E\left(u\left(t_{0}+\tau_{C}, x_{\tau_{C}}\right) \mid \mathcal{N}_{\tau}\right)=E_{x_{0}} u\left(t_{0}+\tau, x_{\tau}\right) .
$$

Here is the statement of the Harnack inequality.

Theorem 6.1. Let $\theta>1$, let $R \in(0, \infty]$, and let $u$ be a nonnegative caloric function in $\bar{C}_{\theta R^{2}, R}$. Then there exists a constant $N$, which depends only on $\theta, \delta,\|b\|$, and $d$, such that

$$
u\left(R^{2}, 0\right) \leq N u(0, x)
$$

whenever $|x| \leq R / 2$. 
Proof. As usual without loss of generality we may concentrate on $R=2$. Then the case of general $\theta>1$ is reduced to that of $\theta \geq 2$ by appropriate change of the time variable $t \rightarrow \tau(t)$. One more observation that the best constant $N$ is obviously decreasing in $\theta$, allows us to restrict our attention to the case of $\theta=2$.

In case $R=\theta=2$, to exclude a trivial situation, additionally assume that

$$
u(4,0)>0 \text {. }
$$

For $\kappa=1 / 2, \eta=1 / 2$, we take $N$ and $\nu$ from Theorem 3.9, call this $N$ $N_{1}$, and, having in mind Theorem 3.8, find $\gamma \in(0,1)$ close to 1 and $\varepsilon>0$ close to zero, for which

$$
1-\varepsilon \geq q(\gamma) 2^{-1}+[1-q(\gamma)] 2^{\nu}
$$

Next, for $r \in[0,1)$, introduce

$$
\mu(r)=u(4,0)(1-r)^{-\nu}, \quad n(r)=\sup \left\{u, \bar{C}_{r}(4,0)\right\} \quad\left(\bar{C}_{0}(4,0):=\{(4,0)\}\right),
$$

and define $r_{0}$ as the greatest number in $r \in[0,1)$ satisfying

$$
n(r) \geq \mu(r) .
$$

Such a number does exist because $n(0)=\mu(0), \mu(r) \rightarrow \infty$ as $r \uparrow 1$, and $n(r)$ is bounded, increasing, and right continuous. Choose $\left(t^{\varepsilon}, x^{\varepsilon}\right) \in \bar{C}_{r_{0}}(4,0)$ such that $n\left(r_{0}\right) \leq(1+\varepsilon) u\left(t^{\varepsilon}, x^{\varepsilon}\right)$ and consider the cylinder

$$
Q:=\left\{(t, x): 0 \leq t-t^{\varepsilon}<\frac{\left(1-r_{0}\right)^{2}}{4}, \quad\left|x-x^{\varepsilon}\right|<\frac{1-r_{0}}{2}\right\} .
$$

As is easy to see $\bar{Q} \subset \bar{C}_{r_{1}}(4,0)$, where $r_{1}=\left(1+r_{0}\right) / 2$. By the definition of $r_{0}$, this implies that

$$
\sup _{\bar{Q}} u<\mu\left(r_{1}\right)=u(4,0)\left(\frac{1-r_{0}}{2}\right)^{-\nu} \leq 2^{\nu} n\left(r_{0}\right) .
$$

We claim that owing to this and (6.2),

$$
\left|Q \cap\left\{u>n\left(r_{0}\right) / 2\right\}\right| \geq(1-\gamma)|Q| .
$$

To argue by contradiction, assume (6.3) is false. Then

$$
\left|Q \cap\left\{u \leq n\left(r_{0}\right) / 2\right\}\right|>\gamma|Q|
$$

and there is a closed set $\Gamma \subset Q \cap\left\{u \leq n\left(r_{0}\right) / 2\right\}$ such that $|\Gamma|>\gamma|Q|$. Introduce $\tau_{\Gamma}$ as the first time the process $\left(t^{\varepsilon}+t, x_{t}\right)$ hits $\Gamma$ and $\tau_{Q}$ as the first time it exits from $Q$. It follows by Remark 6.1 and Theorem 3.8 that (note that $n\left(r_{0}\right) / 2 \leq \sup _{\bar{Q}} u$ )

$$
\begin{gathered}
u\left(t^{\varepsilon}, x^{\varepsilon}\right)=E_{x^{\varepsilon}} I_{\tau_{\Gamma}<\tau_{Q}} u\left(t^{\varepsilon}+\tau_{\Gamma}, x_{\tau_{\Gamma}}\right)+E_{x^{\varepsilon}} I_{\tau_{\Gamma} \geq \tau_{Q}} u\left(t^{\varepsilon}+\tau_{Q}, x_{\tau_{Q}}\right) \\
\leq P_{x^{\varepsilon}}\left(\tau_{\Gamma}<\tau_{Q}\right) n\left(r_{0}\right) / 2+\left(1-P_{x^{\varepsilon}}\left(\tau_{\Gamma}<\tau_{Q}\right)\right) \sup _{\bar{Q}} u \\
\leq q(\gamma) n\left(r_{0}\right) / 2+(1-q(\gamma)) \sup _{\bar{Q}} u \\
\leq q(\gamma) n\left(r_{0}\right) / 2+(1-q(\gamma)) 2^{\nu} n\left(r_{0}\right) .
\end{gathered}
$$


We now have

$$
n\left(r_{0}\right) \leq(1+\varepsilon) n\left(r_{0}\right)\left[q(\gamma) 2^{-1}+(1-q(\gamma)) 2^{\nu}\right] \leq\left(1-\varepsilon^{2}\right) n\left(r_{0}\right),
$$

which is impossible. This proves (6.3).

Next we apply Theorem 5.9 and get that

$$
u\left(t^{\varepsilon}, x\right) \geq p_{0} n\left(r_{0}\right) 2^{-1}
$$

if $\left|x-x^{\varepsilon}\right| \leq\left(1-r_{0}\right) 4^{-1}$, where $p_{0}=p_{0}(d, \delta,\|b\|, \gamma)>0$. After that it only remains to apply Theorem 3.9 to conclude that for $|x| \leq 1$ we have

$$
u(0, x) \geq \frac{1}{2} p_{0} n\left(r_{0}\right) N_{1}^{-1}\left(\frac{1-r_{0}}{4}\right)^{\nu} \geq 2^{-2 \nu-1} p_{0} N_{1}^{-1} u(4,0) .
$$

The theorem is proved.

Since harmonic function are also caloric we have the following.

Corollary 6.2. Let $R \in(0, \infty]$ and let $u$ be a nonnegative harmonic function in $B_{2 R}$. Then for any $x, y \in B_{R}$ we have $u(x) \leq N u(y)$, where $N=$ $N(d, \delta,\|b\|)$.

Corollary 6.3. Let $R \in(0, \infty]$ and let $u \in W_{d_{0}}^{2}\left(B_{2 R}\right)$ be a nonnegative function satisfying $L u=0$ (a.e.) in $B_{2 R}$. Then for any $x, y \in B_{R}$ we have $u(x) \leq N u(y)$, where $N=N(d, \delta,\|b\|)$.

Indeed, by Theorem 1.3 of [13] Itô's formula is applicable and it shows that $u$ is harmonic in $B_{2 R}$.

In the next part of the section we deal with Hölder norm estimates for harmonic functions and potentials. If $z_{1}=\left(t_{1}, x_{1}\right)$ and $z_{2}=\left(t_{2}, x_{2}\right)$, we define

$$
\rho\left(z_{1}, z_{2}\right)=\left|x_{1}-x_{2}\right|+\left|t_{1}-t_{2}\right|^{1 / 2}
$$

and call $\rho\left(z_{1}, z_{2}\right)$ the parabolic distance between $z_{1}$ and $z_{2}$.

Lemma 6.4. Let $R \in(0, \infty]$ and let $u$ be a caloric function in $\bar{C}_{2 R}$. Then there exist constants $N$ and

$$
\alpha_{0} \in(0,1),
$$

depending only on $\delta, d,\|b\|$, such that, for any $\alpha \in\left(0, \alpha_{0}\right]$ and $z_{1}, z_{2} \in C_{R}$, we have

$$
\left|u\left(z_{1}\right)-u\left(z_{2}\right)\right| \leq N R^{-\alpha} \rho^{\alpha}\left(z_{1}, z_{2}\right) \sup \left(|u|, \bar{C}_{2 R}\right) .
$$

Furthermore, $\sup \left(|u|, \bar{C}_{2 R}\right)$ in (6.5) can be replaced by $\operatorname{osc}\left(u, \bar{C}_{2 R}\right)$, where we use the notation

$$
\operatorname{osc}(g, \Gamma)=\underset{\Gamma}{\operatorname{osc}} g=\sup _{\Gamma} g-\inf _{\Gamma} g .
$$

Proof. The case that $R=\infty$ is obtained by passing to the limit and the case $R \in(0, \infty)$ reduces to $R=1$ by using self-similarity. In that case for $r \in(0,2]$, set

$$
w(r)=\operatorname{osc}\left(u, \bar{C}_{r}\right), \quad m(r)=\inf _{\bar{C}_{r}} u, \quad M(r)=\sup _{\bar{C}_{r}} u,
$$




$$
\mu(r)=(1 / 2)(m(r)+M(r)) .
$$

Take $r \leq 1 / 2$ and suppose that

$$
\left|C_{2 r} \cap\{u \leq \mu(r)\}\right| \geq(1 / 2)\left|C_{2 r}\right| .
$$

Then there is a closed $\Gamma \subset C_{2 r} \cap\{u \leq \mu(r)\}$ such that

$$
\left|C_{3 r^{2}, r}\left(r^{2}, 0\right) \cap \Gamma\right| \geq(1 / 4)\left|C_{3 r^{2}, r}\right|
$$

By Theorem 5.9 for any $\left(t_{0}, x_{0}\right) \in \bar{C}_{r}$ we have

$$
P_{x_{0}}\left(\tau_{\Gamma}<\tau_{2 r}\right) \geq p_{0},
$$

where $p_{0}>0$ depends only on $\delta, d,\|b\|, \tau_{\Gamma}$ is the first time $\left(t_{0}+t, x_{t}\right)$ hits $\Gamma$, $\tau_{2 r}$ is its first exit time from $C_{2 r}$. Then by Remark 6.1 for $\tau=\tau_{\Gamma} \wedge \tau_{2 r}$

$$
\begin{gathered}
u\left(t_{0}, x_{0}\right)=E_{x_{0}} u\left(t_{0}+\tau, x_{\tau}\right) . \\
=E_{x_{0}} u\left(t_{0}+\tau_{\Gamma}, x_{\tau_{\Gamma}}\right) I_{\tau_{\Gamma}<\tau_{2 r}}+E_{x_{0}} u\left(t_{0}+\tau_{2 r}, x_{\tau_{2 r}}\right) I_{\tau_{\Gamma} \geq \tau_{2 r}} \\
\leq \mu(r) p_{0}+M(2 r)\left(1-p_{0}\right)
\end{gathered}
$$

(we used that $\mu(r) \leq M(2 r))$. It follows that

$$
\begin{gathered}
M(r) \leq p_{0} \frac{1}{2}(m(r)+M(r))+\left(1-p_{0}\right) M(2 r), \\
\left(1-\frac{p_{0}}{2}\right) M(r) \leq \frac{p_{0}}{2} m(r)+\left(1-p_{0}\right) M(2 r) .
\end{gathered}
$$

Adding to this the obvious inequality

$$
\left(\frac{p_{0}}{2}-1\right) m(r) \leq-\frac{p_{0}}{2} m(r)+\left(p_{0}-1\right) m(2 r)
$$

we get

$$
\left(1-\frac{p_{0}}{2}\right) w(r) \leq\left(1-p_{0}\right) w(2 r), \quad w(r) \leq \varepsilon w(2 r),
$$

where $\varepsilon<1, \varepsilon=\varepsilon(d, K, \delta)$. We may, certainly, assume that $\varepsilon>1 / 2$.

We have proved (6.7) assuming that (6.6) is true. However if (6.6) is false, then $-u$ satisfies an inequality similar to (6.6) and this leads to (6.7) again.

Therefore, $w(r) \leq \varepsilon w(2 r)$ for all $r \leq 1 / 2$. Iterations then yield

$$
w(r) \leq \varepsilon^{2} w(4 r) \quad \text { for } \quad r \leq 1 / 4, \ldots, w(r) \leq \varepsilon^{n} w\left(2^{n} r\right) \quad \text { for } \quad r \leq 2^{-n} .
$$

If $r \leq 1 / 2$ and we take $n:=\left\lfloor-\log _{2} r\right\rfloor$, then $r \leq 2^{-n}$ and

$$
w(r) \leq \varepsilon^{n} w\left(2^{n} r\right) \leq \varepsilon^{-1} r^{\alpha} w(1) \leq 2 \varepsilon^{-1} r^{\alpha} \sup \left(|u|, \bar{C}_{1}\right),
$$

where $\alpha=-\log _{2} \varepsilon \in(0,1)$. This provides an estimate of the oscillation of $u$ in any $C_{r}$ with $r \leq 1 / 2$. The same estimate obviously holds for the oscillation of $u$ in any $C_{r}(t, x) \subset C_{2}$ as long as $r \leq 1 / 2$.

Now take $z_{1}=\left(t_{1}, x_{1}\right), z_{2}=\left(t_{2}, x_{2}\right) \in C_{1}$ such that $r:=\rho\left(z_{1}, z_{2}\right) \leq 1 / 2$ and define

$$
t=t_{1} \wedge t_{2}, \quad x=\left(x_{1}+x_{2}\right) / 2
$$


Then we have $z_{i} \in \bar{C}_{R}(t, x), i=1,2$, and

$$
\begin{aligned}
\left|u\left(z_{1}\right)-u\left(z_{2}\right)\right| & \leq 2 \varepsilon^{-1} r^{\alpha} \sup \left(|u|, \bar{C}_{1}(t, x)\right) \\
& \leq 2 \varepsilon^{-1} \rho^{\alpha}\left(z_{1}, z_{2}\right) \sup \left(|u|, \bar{C}_{2}\right) .
\end{aligned}
$$

In the case that $\rho\left(z_{1}, z_{2}\right) \geq 1 / 2$ we have

$$
\begin{aligned}
\left|u\left(z_{1}\right)-u\left(z_{2}\right)\right| & \leq 2 \sup \left(|u|, \bar{C}_{2}\right) \\
& \leq 2^{1+\alpha} \rho^{\alpha}\left(z_{1}, z_{2}\right) \sup \left(|u|, \bar{C}_{2}\right) .
\end{aligned}
$$

Thus, $N=2^{1+\alpha}+2 \varepsilon^{-1}$ in (6.5) is always a good choice with $R=1$ and $\alpha=\alpha(\delta, d)$ found above. One can take any smaller $\alpha$ as well since $\rho\left(z_{1}, z_{2}\right) \leq N(d) R$. The lemma is proved.

Theorem 6.5. Let $R \in(0, \infty), p \geq d_{0}, p>d / 2+1$, let $g$ be a Borel bounded function on $\bar{C}_{2 R}$ and $f \in L_{p}\left(C_{2 R}\right)$. For $\left(t_{0}, x_{0}\right) \in C_{2 R}$ define

$$
u\left(t_{0}, x_{0}\right)=E_{x_{0}} \int_{0}^{\gamma_{2 R}} f\left(t_{0}+t, x_{t}\right) d t+E_{x_{0}} g\left(t_{0}+\gamma_{2 R}, x_{\gamma_{2 R}}\right),
$$

where $\gamma_{2 R}$ is the first exit time of $\left(t_{0}+t, x_{t}\right)$ from $C_{2 R}$. Then there exists a constant $N$, which depends only on $p, d,\|b\|$, and $\delta$, such that

$$
\left|u\left(z_{1}\right)-u\left(z_{2}\right)\right| \leq N\left(R^{-\alpha} \rho^{\alpha}\left(z_{1}, z_{2}\right) \sup _{\bar{C}_{2 R}}|g|+R^{2-(d+2) / p}\|f\|_{L_{p}\left(C_{2 R}\right)}\right)
$$

for $z_{1}, z_{2} \in C_{R}$ and $\alpha \in\left(0, \alpha_{0}\right]$.

Proof. Parabolic scalings allow us to only concentrate on the case that $R=1$. After that it only remains to observe that $h\left(t_{0}, x_{0}\right):=E_{x_{0}} g\left(t_{0}+\right.$ $\left.\gamma_{2 R}, x_{\gamma_{2 R}}\right)$ is a caloric function, to which Lemma 6.4 is applicable, and $u\left(t_{0}, x_{0}\right)-h\left(t_{0}, x_{0}\right)$ is estimated by Corollary 4.9. The theorem is proved.

Here is a version of Theorem 6.5 which sometimes is slightly more convenient.

Theorem 6.6. Under the conditions of Theorem 6.5 there exists a constant $N$, which depends only on $p, d,\|b\|$, and $\delta$, such that

$$
\left|u\left(z_{1}\right)-u\left(z_{2}\right)\right| \leq N R^{-\beta} \rho^{\beta}\left(z_{1}, z_{2}\right)\left(\sup _{\bar{C}_{2 R}}|u|+R^{2-(d+2) / p}\|f\|_{L_{p}\left(C_{2 R}\right)}\right)
$$

for $z_{1}, z_{2} \in C_{R}$, where

$$
\beta=\frac{\alpha(2 p-d-2)}{\alpha p+2 p-d-2}
$$

and $\alpha=\alpha_{0}(\delta, d)$ is the constant from Theorem 6.5 (or Lemma 6.4).

Proof. Fix $z_{1}, z_{2} \in C_{R}$. Since there is the sup norm of $u$ on the right, it suffices to prove (6.9) assuming that

$$
\xi:=\left(\frac{R}{\rho\left(z_{1}, z_{2}\right)}\right)^{\beta / \alpha} \geq 4
$$

Then set

$$
\bar{R}=\xi \rho\left(z_{1}, z_{2}\right)
$$


If $z_{i}=\left(t_{i}, x_{i}\right), i=1,2$, without losing generality we may assume that $t_{1} \leq t_{2}$. Then for

$$
\left|x_{1}\right|+\bar{R} \leq 2 R \quad \text { and } \quad t_{1}+\bar{R}^{2} \leq 4 R^{2}
$$

we have

$$
z_{1}, z_{2} \in \bar{C}_{\bar{R} / 4}\left(z_{1}\right) \subset \bar{C}_{\bar{R}}\left(z_{1}\right) \subset \bar{C}_{2 R}
$$

Since $z_{1} \in \bar{C}_{R}$, we have $\left|x_{1}\right| \leq R$ and $t_{1} \leq R^{2}$ and, for any of the inequalities (6.10) to go wrong, we have to have $\bar{R} \geq R$, that is,

$$
\left(\frac{R}{\rho\left(z_{1}, z_{2}\right)}\right)^{\beta / \alpha-1} \geq 1
$$

which is only possible if $\rho\left(z_{1}, z_{2}\right) \geq R$ when (6.9) holds trivially with $N=2$. Therefore, in what follows we assume (6.11) and that $\bar{R} \leq R$.

Then by Theorem 6.5 applied to $C_{\bar{R}}\left(z_{1}\right)$ in place of $C_{R}$ we obtain

$$
\left|u\left(z_{1}\right)-u\left(z_{2}\right)\right| \leq N\left(\bar{R}^{-\alpha} \rho^{\alpha}\left(z_{1}, z_{2}\right) \sup _{C_{2 R}}|u|+\bar{R}^{2-(d+2) / p}\|f\|_{L_{p}\left(C_{2 R}\right)}\right),
$$

where the right-hand side is transformed to that of (6.9) by simple arithmetics. The theorem is proved.

Corollary 6.7. Let $R \in(0, \infty), p \geq d_{0}, p>d / 2+1$, and let $u \in W_{p}^{1,2}\left(C_{2 R}\right)$. Define $f=\partial_{t} u+L u$. Then there exists a constant $N$, which depends only on $p, d,\|b\|$, and $\delta$, such that (6.9) holds for $z_{1}, z_{2} \in C_{R}$ with the same $\beta$ as in $(6.9)$.

To prove this it suffices to follow the path laid down in the proof of Theorem 4.13.

In the time-homogeneous situation we have a similar result.

Theorem 6.8. Let $R \in(0, \infty), p \geq d_{0}$, let $g$ be a Borel bounded function on $\bar{B}_{2 R}$ and $f \in L_{p}\left(B_{2 R}\right)$. For $x_{0} \in B_{2 R}$ define

$$
u\left(x_{0}\right)=E_{x_{0}} \int_{0}^{\tau_{2 R}} f\left(x_{t}\right) d t+E_{x_{0}} g\left(x_{\tau_{2 R}}\right),
$$

(recall that $\tau_{2 R}$ is the first exit time of $x_{t}$ from $B_{2 R}$ ). Then there exists a constant $N$, which depends only on $p, d,\|b\|$, and $\delta$, such that

$$
\left|u\left(x_{1}\right)-u\left(x_{2}\right)\right| \leq N R^{-\alpha}\left|x_{1}-x_{2}\right|^{\alpha}\left(\sup _{\bar{B}_{2 R}}|u|+R^{2-d / p}\|f\|_{L_{p}\left(B_{2 R}\right)}\right)
$$

for $x_{1}, x_{2} \in B_{R}$ and $\alpha=\alpha(d, \delta,\|b\|) \in(0,1)$.

This theorem is proved in the same way as Theorem 6.6 by using the fact that $h\left(x_{0}\right):=E_{x_{0}} g\left(x_{\tau_{2 R}}\right)$ is a caloric function, to which Lemma 6.4 is applicable, and $u-h$ admits an estimate by Theorem 3.2.

Similarly to Corollary 6.7 we have the following corollary of Theorem 6.8.

Corollary 6.9. Let $R \in(0, \infty), p \geq d_{0}$, and let $u \in W_{p}^{2}\left(B_{2 R}\right)$. Define $f=L u$. Then there exists a constant $N$, which depends only on $p, d,\|b\|$, and $\delta$, such that (6.12) holds for $x_{1}, x_{2} \in B_{R}$ with the same $\alpha$ as in (6.12). 
We finish the paper by proving a result showing that the function $u$ from Theorem 6.8 is an $L_{d_{0}}$-viscosity solution of the equation $L u=-f$ in $B_{2 R}$.

Theorem 6.10. Let $u$ be as in Theorem 6.8. Then for any $\phi \in W_{d_{0}}^{2}\left(B_{2 R}\right)$ and any point $x_{0} \in B_{2 R}$ at which $u-\phi$ has local maximum we have

$$
\lim _{\varepsilon \downarrow 0} \operatorname{esssup}(L \phi+f) \geq 0 .
$$

The proof of this theorem is based on the following.

Lemma 6.11. There is a constant $N=N(d, \delta,\|b\|)$ such that for any $B_{r}(x)$ satisfying $\bar{B}_{r}(x) \subset B_{2 R}$ and $\phi \in W_{d_{0}}^{2}\left(B_{r}(x)\right)$ we have on $B_{r}(x)$ that

$$
u \leq \phi+N r^{2-d / d_{0}}\left\|(L \phi+f)_{+}\right\|_{L_{d_{0}}\left(B_{r}(x)\right)}+\max _{\partial B_{r}(x)}(u-\phi)_{+} \cdot
$$

Proof. For $x_{0} \in B_{r}(x)$ by strong Markov property, with $\tau$ defined as the first exit time of $x_{t}$ from $B_{r}(x)$, and Itô's formula we have

Hence,

$$
\begin{gathered}
u\left(x_{0}\right)=E_{x_{0}}\left(\int_{0}^{\tau} f\left(x_{t}\right) d t+u\left(x_{\tau}\right)\right), \\
\phi\left(x_{0}\right)=E_{x_{0}}\left(\int_{0}^{\tau}(-L \phi)\left(x_{t}\right) d t+\phi\left(x_{\tau}\right)\right) .
\end{gathered}
$$

$$
u\left(x_{0}\right)-\phi\left(x_{0}\right) \leq E_{x_{0}}\left(\int_{0}^{\tau}(L \phi+f)_{+}\left(x_{t}\right) d t+(u-\phi)_{+}\left(x_{\tau}\right)\right)
$$

and (6.14) follows from Theorem 3.2. The lemma is proved.

Proof of Theorem 6.10. Let $x_{0} \in B_{2 R}$ be a point at which $u-\phi$ has local maximum. Then for $\varepsilon>0$ and all small $r>0$ for

$$
\phi_{\varepsilon, r}(x)=\phi(x)-\phi\left(x_{0}\right)+u\left(x_{0}\right)+\varepsilon\left(\left|x-x_{0}\right|^{2}-r^{2}\right)
$$

we have that

$$
\max _{\partial B_{r}\left(x_{0}\right)}\left(u-\phi_{\varepsilon, r}\right)_{+}=0
$$

Hence, by Lemma 6.11

$$
\varepsilon r^{2}=\left(u-\phi_{\varepsilon, r}\right)\left(x_{0}\right) \leq N_{1} r^{2-d / d_{0}}\left\|\left(L \phi_{\varepsilon}+f\right)_{+}\right\|_{L_{d_{0}}\left(B_{r}\left(x_{0}\right)\right)},
$$

where $\phi_{\varepsilon}=\phi+\varepsilon\left|x-x_{0}\right|^{2}$. Here $\left(L \phi_{\varepsilon}+f\right)_{+} \leq(L \phi+f+2 \varepsilon \operatorname{tr} a)_{+}+N \varepsilon r|b|$ and in light of Hölder's inequality

$$
\begin{gathered}
\left\|\left(L \phi_{\varepsilon}+f\right)_{+}\right\|_{L_{d_{0}}\left(B_{r}\left(x_{0}\right)\right)} \leq\left\|(L \phi+f+2 \varepsilon \operatorname{tr} a)_{+}\right\|_{L_{d_{0}}\left(B_{r}\left(x_{0}\right)\right)} \\
+N_{2} \varepsilon r^{d / d_{0}}\|b\|_{L_{d}\left(B_{r}\left(x_{0}\right)\right) .}
\end{gathered}
$$

Here the last term multiplied by $N_{1} r^{2-d / d_{0}}$ is smaller than $(1 / 2) \varepsilon r^{2}$ for all sufficiently small $r$ (depending on how fast $\|b\|_{L_{d}\left(B_{r}\left(x_{0}\right)\right)} \rightarrow 0$ ). Therefore, for such $r$

$$
(1 / 2) \varepsilon r^{2}=\left(u-\phi_{\varepsilon, r}\right)\left(x_{0}\right) \leq N r^{2-d / d_{0}}\left\|(L \phi+f+2 \varepsilon \operatorname{tr} a)_{+}\right\|_{L_{d_{0}}\left(B_{r}\left(x_{0}\right)\right)}
$$




$$
\begin{aligned}
\leq & N r^{2} \operatorname{esssup}_{\left.B_{r}\left(x_{0}\right)\right)}(L \phi+f+2 \varepsilon \operatorname{tr} a)_{+}, \\
& \quad \operatorname{esssup}_{\left.B_{r}\left(x_{0}\right)\right)}(L \phi+f+2 \varepsilon \operatorname{tr} a)>0,
\end{aligned}
$$

and the last relation implies (6.13) after setting $r, \varepsilon \downarrow 0$. The theorem is proved.

Remark 6.2. Let $D$ be a bounded domain in $\mathbb{R}^{d}, g$ be a Borel bounded function on $\mathbb{R}^{d}$, and $f \in L_{d_{0}}(D)$. Introduce

$$
u(x)=E_{x}\left(\int_{0}^{\tau} f\left(x_{t}\right) d t+g\left(x_{\tau}\right)\right)
$$

where $\tau$ is the first exit time of $x_{t}$ from $D$. Then by the strong Markov property for any domain $G \subset D$

$$
u(x)=E_{x}\left(\int_{0}^{\gamma} f\left(x_{t}\right) d t+u\left(x_{\gamma}\right)\right)
$$

where $\gamma$ is the first exit time of $x_{t}$ from $G$. Therefore, Theorem 6.10 implies that $u$ is a viscosity solution of $L u+f=0$ in $D$. It is Hölder continuous in $D$ in light of Theorem 6.8.

Its boundary behavior can be investigated by using, for instance, Theorem 4.10 of [13], which says that if $0 \in \partial D$ and for some constants $\rho, \gamma>0$ and any $r \in(0, \rho)$ we have $\left|B_{r} \cap D^{c}\right| \geq \gamma\left|B_{r}\right|$, then there exists $\beta=$ $\beta(d, \delta,\|b\|, \gamma)>0$ such that, for any nonnegative $h \in L_{d_{0}}(D)$ and $x \in D$,

$$
E_{x} \int_{0}^{\tau} h\left(x_{t}\right) d t \leq N|x|^{\beta}\|h\|_{L_{d_{0}}(D)},
$$

where $N$ depends only on $d, \delta,\|b\|, \gamma, \rho$, and the diameter of $D$.

The reader can find numerous properties of $L_{p}$-viscosity solutions in elliptic and parabolic settings in articles initiated by [4], references to many of them can be found in [11].

Acknowledgment. The author thanks T. Yastrzhembskiy for pointing out several mistakes and misprints in the first draft of the paper.

\section{REFERENCES}

[1] S.V. Anulova and G. Pragarauskas, Weak Markov solutions of stochastic equations, Litovsk. Mat. Sb., Vol. 17 (1977), No. 2, 5-26, in Russian; English translation: Lithuanian Math. J., Vol. 17 (1977), No. 2, 141-155.

[2] R. Bass, "Diffusions and elliptic operators", Probability and its Applications, Springer-Verlag, New York, 1998.

[3] R.M. Blumenthal and R.K. Getoor, "Markov processes and potential theory", Pure and Applied Mathematics, A Series of Monographs and Textbooks, Vol. 29, Academic Press, New York and London, 1968.

[4] L. Caffarelli, M. G. Crandall, M. Kocan, and A. Święch,, On viscosity solutions of fully nonlinear equations with measurable ingredients, Comm. Pure Appl. Math., Vol. 49 (1996), No. 4, 365-397. 
[5] E. B. Dynkin, "Markov processes", Fizmatgiz, Moscow, 1963 in Russian: English translation in Grundlehren Math. Wiss., Vols. 121, 122, Springer-Verlag, Berlin, 1965.

[6] E.B. Fabes and D.W. Stroock, The $L^{p}$-integrability of Green's functions and fundamental solutions for elliptic and parabolic equations, Duke Math. J., Vol. 51 (1984), No. 4, 997-1016.

[7] N.V. Krylov, On the selection of a Markov process from a system of processes and the construction of quasi-diffusion processes, Izvestiya Akademii Nauk SSSR, seriya matematicheskaya, Vol. 37 (1973), No. 3, 691-708 in Russian; English translation in Math. USSR Izvestija, Vol. 7 (1973), No. 3, 691-709.

[8] N.V. Krylov, Some estimates for the density of the distribution of a stochastic integral, Izvestiya Akademii Nauk SSSR, seriya matematicheskaya, Vol. 38 (1974), No. 1, 228-248 in Russian; English translation in Math. USSR Izvestija, Vol. 8 (1974), No. 1, 233-254.

[9] N.V. Krylov, "Controlled diffusion processes", Nauka, Moscow, 1977 in Russian; English transl. Springer, 1980.

[10] N.V. Krylov, "Introduction to the theory of diffusion processes", Amer. Math. Soc., Providence, RI, 1995.

[11] N.V. Krylov, "Sobolev and viscosity solutions for fully nonlinear elliptic and parabolic equations", Mathematical Surveys and Monographs, 233, Amer. Math. Soc., Providence, RI, 2018.

[12] N.V. Krylov, On stochastic Itô processes with drift in $L_{d}$, http://arxiv.org/abs/2001.03660

[13] N.V. Krylov, On stochastic equations with drift in $L_{d}$, http://arxiv.org/abs/2001.04008

[14] N.V. Krylov and M.V. Safonov, A certain property of solutions of parabolic equations with measurable coefficients, Izvestiya Akademii Nauk SSSR, seriya matematicheskaya, Vol. 44 (1980), No. 1, 161-175 in Russian; English translation in Math. USSR Izvestija, Vol. 16 (1981), No. 1, 151-164.

[15] P.-L. Lions, Some recent results in the optimal control of diffusion processes, Stochastic analysis (Katata/Kyoto, 1982), pp 333-367, North-Holland Math. Library, 32, North-Holland, Amsterdam, 1984.

[16] N. I. Portenko, "Generalized diffusion processes", Nauka, Moscow, 1982 in Russian; English translation: Amer. Math. Soc. Providence, Rhode Island, 1990.

[17] M. V. Safonov, Harnack inequalities for elliptic equations and Hölder continuity of their solutions, Zap. Nauchn. Sem. Leningrad. Otdel. Mat. Inst. Steklov (LOMI), Vol. 96 (1980), 272-287 in Russian; English transl. in Journal of Soviet Mathematics, Vol. 21 (March 1983), No. 5, 851-863.

[18] M.V. Safonov, Non-divergence elliptic equations of second order with unbounded drift, Nonlinear partial differential equations and related topics, 211-232, Amer. Math. Soc. Transl. Ser. 2, 229, Adv. Math. Sci., 64, Amer. Math. Soc., Providence, RI, 2010.

[19] D.W. Stroock and S.R.S. Varadhan "Multidimensional diffusion processes", Grundlehren Math. Wiss., Vol. 233, Springer-Verlag, Berlin and New York, 1979.

[20] T. Yastrzhembskiy, A note on the strong Feller property of diffusion processes, preprint.

[21] Xicheng Zhang and Guohuan Zhao, Stochastic Lagrangian path for Leray solutions of $3 d$ Navier-Stokes equations, preprint.

E-mail address: nkrylov@umn.edu

127 Vincent Hall, University of Minnesota, Minneapolis, MN, 55455 